\title{
SECOND-ORDER TOPOLOGICAL EXPANSION FOR ELECTRICAL IMPEDANCE TOMOGRAPHY
}

\author{
M. HINTERMÜLLER*, A. LAURAIN ${ }^{\dagger}$, AND A.A. NOVOTNY ${ }^{\ddagger}$
}

\begin{abstract}
Second-order topological expansions in electrical impedance tomography problems with piecewise constant conductivities are considered. First-order expansions usually consist of local terms typically involving the state and the adjoint solutions and their gradients estimated at the point where the topological perturbation is performed. In the case of second-order topological expansions, non-local terms which have a higher computational cost appear. Interactions between several simultaneous perturbations are also considered. The study is aimed at determining the relevance of these non-local and interaction terms from a numerical point of view. A level set based shape algorithm is proposed and initialized by using topological sensitivity analysis.
\end{abstract}

AMS subject classifications. 49Q10, 49K24, 49N45

Key words. electrical impedance tomography, inverse problem, shape and topological derivative, level sets

1. Introduction. Electrical Impedance Tomography (EIT) is a non-destructive imaging technique which has various applications in medical imaging, geophysics and other fields; see [5] and the references therein. Its purpose is to reconstruct the electric conductivity and permittivity of hidden objects inside a medium with the help of boundary field measurements. If we denote by $\Omega$ the background medium with $\Sigma$ its smooth boundary where the currents are applied, then the commonly used continuum model is

$$
-\operatorname{div}\left(q_{\omega} \nabla u\right)=0 \text { in } \Omega, \quad q_{\omega} \partial_{n} u=f_{\omega} \text { on } \Sigma .
$$

Here, $u=u_{\omega}$ is the electric potential or voltage, and the admittivity $q_{\omega}$ is given by $q_{\omega}(x)=$ $\sigma_{\omega}(x)+i \omega \varepsilon_{\omega}(x)$, where $\sigma_{\omega}$ is the electric conductivity, $\varepsilon_{\omega}$ is the electric permittivity, $\omega$ is the angular frequency of the applied current, and $x \in \Omega$. We also need the conservationof-charge-condition $\int_{\Sigma} f_{\omega}=0$ and the condition $\int_{\Sigma} u_{\omega}=0$, which amounts to choosing a "ground" or reference voltage. For further extensions of this continuum model in case of real experiments, we refer to the survey papers $[5,8]$ and the references therein.

A widely used solution approach to the inverse problem of identifying $q$ from given measurement data is to minimize the $L_{2}$-distance between the potentials $u_{k}$ pertinent to a certain given number $M$ of applied currents $f_{k}$ and corresponding boundary measurements $m_{k}$. Since the problem is known to be severely ill-posed, it is necessary to add a regularization term to the least-squares term in the objective functional. The resulting minimization problem then becomes

$$
\min _{q} \sum_{k=1}^{M} \int_{\Sigma}\left(u_{k}(q)-m_{k}\right)^{2}+\beta \int_{\Omega}|\nabla q|
$$

where the first term takes care of matching the given measurements and the second term implements the regularization with a positive parameter $\beta$. Here, $u_{k}(q)$ denotes the solution of (1.1) with $f=f_{k}$. The nondifferentiable nature of the regularization term is well-known to preserve discontinuities, i.e., the interfaces between the background and the inclusions [9].

Several algorithms have been proposed for solving particular situations containing additional information on the data or the underlying configuration. For instance, in $[2,6,7]$

\footnotetext{
${ }^{*}$ Humboldt-University of Berlin, Department of Mathematics, Berlin, Germany, and University of Graz, Department of Mathematics and Scientific Computing, Graz, Austria.

${ }^{\dagger}$ University of Graz, Department of Mathematics and Scientific Computing, Graz, Austria.

${ }^{\ddagger}$ Laboratório Nacional de Computação Científica LNCC/MCT, Petropolis, Brazil.
} 
the background medium is assumed smooth (with known conductivity) and containing a certain number of small inclusions with a higher or lower conductivity. The hypothesis of small inhomogeneities allows to perform an asymptotic analysis which can be used to design different specialized reconstruction algorithms. In the present paper, a similar asymptotic analysis yields the topological derivative, which helps to detect the location of the inclusions. Another possible hypothesis requires the conductivity $q$ to be piecewise constant. In this case, the objects are assumed to have sharp interfaces, thus, enabling a shape optimization approach; see [9] for a related concept using level set functions for representing the inclusions. Therefore we may use the tools of shape and topology optimization $[22,23]$ to determine the number and positions as well as the shape of potential inclusions. Algorithmically one first uses the topological derivative for the EIT-objective providing topological information on the regions with different conductivities. Then, this information is utilized to initialize a shape-sensitivity based procedure for minimizing the least-squares functional in (1.2).

Most algorithms relying on the topological derivative are based on first-order expansions. This usually provides a good initial guess for the subsequent shape-based minimization, and may also be used in an iterative process. However, we have shown in [15] that standard firstorder topological expansions might lead to a wrong initial guess in the case of EIT due to the non-uniform estimates for the remainder. In fact, in the asymptotic expansion of the leastsquares objective, the remainder becomes singular as the trial inclusion comes close to the boundary of $\Omega$. This results in a poor approximation of the objective functional. To overcome this problem, in [15] we considered additional terms in the topological expansion which were concentrated on the boundary of the domain.

In this paper, we extend this perspective by considering applications of higher-order expansions. It has been shown already $[4,10]$ that higher-order topological expansions may enhance the quality of the reconstruction in inverse problems. Higher-order terms in the asymptotic expansion of the steady-state voltage potential were studied in [1]. In case of one inclusion, a higher-order expansion yields a more accurate guess on location and size of the inclusion. In this paper, the case of the simultaneous creation of several inclusions, possibly close to the boundary, is studied along with the derivation of corresponding shape derivatives and their numerical realization in EIT. The pertinent asymptotic expansion provides interesting information on interactions of inclusions. These interactions only appear in the expansion starting from the second-order terms. It is indeed well-known that the first-order term obtained for a set of several inclusions is merely the sum of the first-order terms coming from each inclusion considered separately. Interestingly, the obtained formula for the higheroder topological derivative raises several questions from the algorithmic and theoretical point of view, which could be the topic of further research.

The rest of the paper is organized as follows. In section 2 the EIT problem with a piecewise constant conductivity is described and the corresponding shape functional is introduced. In section 3 the notion of topological derivative for the EIT problem is first defined. Then the asymptotic expansion of the perturbed solution of the EIT problem is performed and used further to obtain the expansion of the shape functional introduced in section 2. In section 4, the shape and conductivity derivatives, which will be used later in our algorithm, are derived. In section 5 a report on numerical tests is given.

2. The EIT problem. In this paper we consider the case where the conductivity $q$ is real, which corresponds to the static regime $\omega=0$ in (1.1). Let $\Omega$ be a bounded domain in $\mathbb{R}^{N}, N \geq 2$, with smooth boundary $\Sigma$. We assume that $\Omega$ contains material with electrical conductivity $q_{\Omega}(x) \geq \bar{q}>0$ for all $x \in \Omega$. Then the electrical potential $u$ satisfies

$$
-\operatorname{div}\left(q_{\Omega} \nabla u\right)=0 \text { in } \Omega, \quad q_{\Omega} \partial_{n} u=f \text { on } \Sigma,
$$


where $f \in H^{-1 / 2}(\Sigma)$ is an applied current density on $\Sigma$ satisfying the conservation of charge $\langle f, 1\rangle_{H^{-1 / 2}(\Sigma), H^{1 / 2}(\Sigma)}=0$. The EIT problem consists in finding the electrical conductivity $q_{\Omega}$ inside $\Omega$ using a set of given values of applied current densities $f_{k}, k=1, \ldots, M$ on $\Sigma$, where $M$ is a positive integer, and the corresponding electrical potentials $u_{k}$ on $\Sigma$.

Here we assume that the conductivity is piecewise constant, i.e., there are a background conductivity $q_{0}>0$ and an unknown number $n_{q}$ of inclusions with conductivities $q_{i}, i=$ $1, . ., n_{q}$. Then $\Omega$ can be split into $n_{q}+1$ disjoint domains $\Omega_{0}$ and $\Omega_{i}$, with conductivities $q_{0}$ and $q_{i}, i=1, . ., n_{q}$, respectively. This yields $\Omega=\bigcup_{i=0}^{n_{q}} \Omega_{i}$ with $\Sigma \cap \Gamma_{i}=\emptyset$, where $\Gamma_{i}=\partial \Omega_{i}$. We then have $q_{\Omega}=\sum_{i=0}^{n_{q}} q_{i} \mathbb{1}_{\Omega_{i}}$. Due to the particular form of $q$ the regularization term becomes $\int_{\Omega}\left|\nabla q_{\Omega}\right|=\sum_{i=1}^{n_{q}}\left|q_{0}-q_{i}\right| \mathcal{P}\left(\Gamma_{i}\right)$, where $\mathcal{P}\left(\Gamma_{i}\right)$ stands for the perimeter of $\Omega_{i}$. Therefore, the EIT problem is reduced to solving the following minimization problem which depends only on $\Omega_{i}$ and the scalar values $q_{i}, i=1, \ldots, n_{q}$ :

$$
\operatorname{minimize} \mathcal{J}\left(\left\{\Omega_{i}, q_{i}\right\}_{i=0}^{n_{q}}\right)=\sum_{k=1}^{M} \int_{\Sigma}\left(u_{k}\left(\left\{\Omega_{i}, q_{i}\right\}_{i=0}^{n_{q}}\right)-m_{k}\right)^{2}+\beta \sum_{i=1}^{n_{q}}\left|q_{0}-q_{i}\right| \mathcal{P}\left(\Gamma_{i}\right),
$$

where $u_{k}$ is the solution of (2.1) with $f_{k}$, a known boundary current density for $k \in\{1, . ., M\}$, on the right-hand side. Further, $m_{k}$ is the boundary measurement corresponding to $f_{k}$. In order to fulfill the compatibility conditions required for the Neumann boundary condition in (2.1), the measurements must satisfy

$$
\int_{\Sigma} m_{k}=0, \quad k=1, . ., M
$$

Since the solution of the Neumann problem (2.1) is not unique, we impose

$$
\int_{\Sigma} u_{k}=0, \quad k=1, . ., M
$$

in order to obtain uniqueness. We also introduce the functional

$$
J\left(\left\{\Omega_{i}\right\}_{i=1}^{n_{q}}\right)=\sum_{k=1}^{M} \int_{\Sigma}\left(u_{k}\left(\left\{\Omega_{i}\right\}_{i=1}^{n_{q}}\right)-m_{k}\right)^{2}+\beta \sum_{i=1}^{n_{q}}\left|q_{0}-q_{i}\right| \mathcal{P}\left(\Gamma_{i}\right),
$$

where the conductivities $q_{i}$ are now assumed to be fixed, while as in (2.2), the functional in (2.5) still contains the unknown quantities $\Omega_{i}, i=1, . ., n_{q}$. Hence, for fixed conductivities $q_{i},(2.2)$ represents a shape optimization problem.

3. Topological derivative. Singular perturbations of domains were first considered theoretically in [16,17] and practically in [12]. This idea was further developed by Sokolowski and Zochowski [22] and Guillaume and Masmoudi [13], by introducing the topological derivative. The topological derivative measures the variation of a cost functional depending on a domain, when a small change in the topology of this domain occurs, for instance through the creation of a small hole of any shape. Let $\Omega$ be an open set in $\mathbb{R}^{N}, B(x, \varepsilon)$ a ball

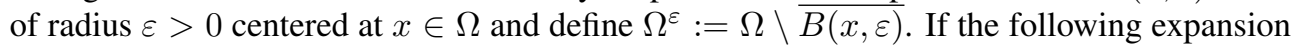
of $J\left(\Omega^{\varepsilon}\right)$ exists

$$
J\left(\Omega^{\varepsilon}\right)=J(\Omega)+\rho(\varepsilon) \mathcal{T}(x)+o(\rho(\varepsilon)),
$$

with $\rho(\varepsilon) \rightarrow 0$ and $o(\rho(\varepsilon)) / \rho(\varepsilon) \rightarrow 0$ as $\varepsilon \rightarrow 0$, then $\mathcal{T}(x)$ is called the topological derivative of $J$ at $x$. We adapt here a notion, which was originally introduced for structural optimization, to the EIT problem. In EIT we do not create "holes" as in structural optimization, but we consider local perturbations $q^{\varepsilon}$ of the conductivity $q$ of the type $q^{\varepsilon}=q_{0}$ in $\Omega^{\varepsilon}$, 
$q^{\varepsilon}=q_{1}$ in $\overline{B(x, \varepsilon)}$, and then we seek an expansion similar to (3.1).

In what follows, for the sake of simplicity, we consider only one measurement, i.e. $M=1$, and write $q$ instead of $q_{0}$ for convenience. We point out that the case of several measurements is readily deduced from the case $M=1$. Now we assume that the domain $\Omega$ is perturbed by introducing several simultaneous inclusions, i.e. $\Omega^{\varepsilon}=\Omega \backslash\left(\cup_{i=1}^{n_{q}} \overline{B_{i}^{\varepsilon}}\right)$ with $B_{i}^{\varepsilon} \cap B_{j}^{\varepsilon}=\varnothing$ for $i \neq j$, where $B_{i}^{\varepsilon}$ is a ball of radius $\varepsilon$ and center $x_{i} \in \Omega$ which is associated with the conductivity $q_{i}$. Therefore, we are in the framework described in section 2, with $\Omega^{\varepsilon}$ and $B_{i}^{\varepsilon}$ corresponding to $\Omega_{0}$ and $\Omega_{i}$ from section 2 , respectively. We consider ball-shaped inclusions for simplicity but inclusions of general shapes can be considered as well; see $[4,6,7,17,18,19]$. Ball-shaped inclusions with different radii may also be considered. The formulas for different radii or shapes are structurally the same as for ball-shaped inclusions, and may be obtained without difficulty, but the numerical cost induced by considering different radii is considerable. This can also be considered as a sub-case of inclusions with different shapes. Therefore it is better to capture these features later with shape sensitivity.

For the topological derivative of $J$, we first study the asymptotic expansion (with respect to $\varepsilon$ ) of the shape functional $J\left(\left\{B_{i}^{\varepsilon}\right\}_{i \in\left\{1, . ., n_{q}\right\}}\right)$ of (2.5). In this framework, we are able to prove that $u_{\varepsilon}$, defined as the solution of (2.1) with $q_{\Omega}=q \mathbb{1}_{\Omega^{\varepsilon}}+\sum_{i=1}^{n_{q}} q_{i} \mathbb{1}_{B_{i}^{\varepsilon}}$, can be written as $u_{\varepsilon}=u^{\varepsilon} \mathbb{1}_{\Omega^{\varepsilon}}+\sum_{i=1}^{n_{q}} u_{i}^{\varepsilon} \mathbb{1}_{B_{i}^{\varepsilon}}$, with $\left(u^{\varepsilon}, u_{i}^{\varepsilon}\right), i=1,2, \ldots, n_{q}$, the solution of the following coupled system

$$
\begin{array}{ll}
-\Delta u^{\varepsilon}=0 \text { in } \Omega^{\varepsilon}, & q \partial_{n} u^{\varepsilon}=f \text { on } \Sigma, \quad q \partial_{n} u^{\varepsilon}=q_{i} \partial_{n} u_{i}^{\varepsilon} \text { on } \partial B_{i}^{\varepsilon}, \\
-\Delta u_{i}^{\varepsilon}=0 \text { in } B_{i}^{\varepsilon}, & u_{i}^{\varepsilon}=u^{\varepsilon} \text { on } \partial B_{i}^{\varepsilon},
\end{array}
$$

Here $n$ stands for the outer unit normal vector to $\partial \Omega^{\varepsilon}$. The normal derivative is denoted $\partial_{n}(\cdot)=\nabla_{x}(\cdot) \cdot n$. The Neumann problem (3.2) is compatible, since we have $\int_{\Sigma} f=0$ by definition and $\int_{\partial B_{i}^{\varepsilon}} q_{i} \partial_{n} u_{i}^{\varepsilon}=-\int_{B_{i}^{\varepsilon}} \Delta u_{i}^{\varepsilon}=0$. As the solution of (3.2) is defined only up to a constant, we impose $\int_{\Sigma} u^{\varepsilon}=0$ to get uniqueness. In order to compute the topological derivative of $J$, we need to perform the asymptotic expansion of $u_{\varepsilon}$, and thus of $u^{\varepsilon}$ and $u_{i}^{\varepsilon}$, as $\varepsilon \rightarrow 0$.

3.1. Preliminary description of the asymptotic procedure. We are looking for an asymptotic expansion of $u^{\varepsilon}$ of the type

$u^{\varepsilon}(x)=u^{0}(x)+\sum_{i=1}^{n_{q}}\left(U_{i}\left(y_{i}\right)+V_{i}\left(y_{i}\right)+W_{i}\left(y_{i}\right)+\sum_{j \neq i}^{n_{q}} \widetilde{U}_{i}^{j}\left(y_{i}\right)\right)+g(x)+\sum_{i=1}^{n_{q}} G_{i}\left(y_{i}\right)+v^{\varepsilon}(x)$,

where $x$ stands for the so-called slow variable in the domain $\Omega$, and $y_{i}:=\varepsilon^{-1}\left(x-x_{i}\right)$ for the fast variable in the unbounded domain $\mathbb{R}^{N} \backslash \overline{B^{1}}$, with $B^{1}$ the ball of radius 1 and center $\mathcal{O}$, the origin of a Cartesian coordinate system. The names fast and slow variables refer to the rapid growth of $y_{i}$ compared to $x$ due to the factor $\varepsilon^{-1}$, which tends to infinity as $\varepsilon \rightarrow 0$, in the definition of $y_{i}$. Here $u^{0}, g$ and $v^{\varepsilon}$ are terms of regular type, whereas $U_{i}, V_{i}, W_{i}$ and $\widetilde{U}_{i}^{j}$ are terms of boundary layer type, which depend on the fast variable $y_{i}$.

The structure of the asymptotic expansion (3.4) comes from general results on asymptotic expansions originally considered in $[16,17]$. In this paper, we use the method of compound asymptotic expansions introduced in [17]. The first term $u^{0}$ of expansion (3.4) is the solution of (3.2)-(3.3) for $\varepsilon=0$ and corresponds to the unperturbed problem. Inserting $u^{0}$ into the singularly perturbed problem (3.2)-(3.3) brings a discrepancy on the inclusions $B_{i}^{\varepsilon}$, which is compensated at the first, second, and third order by the boundary layers $U_{i}, V_{i}$ and $W_{i}$, respectively. When considering only the first-order expansion for the topological derivative, 
it is enough to look for the boundary layer $U_{i}$, whereas it is necessary to go further in the expansion of $u^{\varepsilon}$ for our higher-order asymptotics. When $n_{q}>1$, the boundary layer $U_{i_{1}}$ leaves a discrepancy on the ball $B_{i_{2}}^{\varepsilon}$ for $i_{2} \neq i_{1}$, which is compensated by introducing the boundary layer $\widetilde{U}_{i_{1}}^{i_{2}}$. Therefore, the term $\widetilde{U}_{i}^{j}$ takes into account the interactions between several inclusions, and does not appear when $n_{q}=1$ or when we stick to first-order expansions. This is one of the several interesting features of higher-order expansions.

The boundary layers $U_{i}, V_{i}, W_{i}$ and $\widetilde{U}_{i}^{j}$ leave discrepancies on the outer boundary $\Sigma$, which are compensated by the function of regular type $g$. Then we introduce the boundary layers $G_{i}$ which correct the discrepancies left by $g$ on the balls $B_{i}^{\varepsilon}$. The last term $v^{\varepsilon}$ is the remainder for which estimates can be given. Note that the first terms of the approximation, $U_{i}, V_{i}, W_{i}$ and $\widetilde{U}_{i}^{j}$, are local terms in the sense that they only depend on the unperturbed solution $u^{0}$ and of its derivatives evaluated at $x_{i}$, whereas the last terms of expansion (3.4), starting from $g$, are non-local in the sense that they solve partial differential equations which depend on the positions $x_{i}, i=1, . ., n_{q}$. Although this does not raise any problem from the theoretical point of view, it is apparently an obstacle for numerical realization since the computational cost might be prohibitive. We will see how we can deal with this issue in the numerical results. In the corresponding sections, the main observation is that we may determine functions which depend only on the geometry of $\Omega$. Hence, these functions may be computed "off-line", stored and used later in all subsequent computations.

3.2. Asymptotic expansion of the solution. In what follows, the notation $D^{k} \phi\left(x_{i}\right)(x-$ $\left.x_{i}\right)^{k}$ represents the derivative of order $k$ of a function $\phi$ in direction $x-x_{i}$. Following the description in section 3.1, the first step of the asymptotic expansion is to approximate $u^{\varepsilon}$ by $u^{0}$, the solution of (3.2) for $\varepsilon=0$ :

$$
-\Delta u^{0}=0 \text { in } \Omega, \quad q \partial_{n} u^{0}=f \text { on } \Sigma .
$$

The compatibility condition for (3.5) is satisfied, and the uniqueness of the solution follows from (2.4). Thus, we introduce the rest $\mathcal{R}^{\varepsilon}=u^{\varepsilon}-u^{0}$ such that

$$
u^{\varepsilon}=u^{0}+\mathcal{R}^{\varepsilon} .
$$

Since the solution $u^{0}$ is harmonic in $\Omega$, it is of class $\mathcal{C}^{\infty}$ in the interior of $\Omega$, and we may write the following expansion of $u^{0}$ at $x \in B_{i}^{\varepsilon}$ :

$u^{0}(x)=u^{0}\left(x_{i}\right)+\nabla u^{0}\left(x_{i}\right) \cdot\left(x-x_{i}\right)+\frac{1}{2} D^{2} u^{0}\left(x_{i}\right)\left(x-x_{i}\right)^{2}+\frac{1}{3 !} D^{3} u^{0}\left(x_{i}\right)\left(x-x_{i}\right)^{3}+\mathcal{S}_{i}^{u}(x)$,

where the remainder $\mathcal{S}_{i}^{u}$ is such that $\mathcal{S}_{i}^{u}(x)=(4 !)^{-1} D^{4} u^{0}\left(\xi_{i}\right)\left(x-x_{i}\right)^{4}$ with $\xi_{i} \in\left[x, x_{i}\right]$. Plugging (3.6) and (3.7) into the Dirichlet conditions in (3.3), and considering that $u^{0}$ is harmonic in $\Omega$, we get

$$
\begin{aligned}
u_{i}^{\varepsilon}(x) & =u^{0}\left(x_{i}\right)+\nabla u^{0}\left(x_{i}\right) \cdot\left(x-x_{i}\right) \\
& +\frac{1}{2} D^{2} u^{0}\left(x_{i}\right)\left(x-x_{i}\right)^{2}+\frac{1}{3 !} D^{3} u^{0}\left(x_{i}\right)\left(x-x_{i}\right)^{3}+\mathcal{S}_{i}^{u}(x)+v_{i}^{\varepsilon}(x),
\end{aligned}
$$

where the quantity $v_{i}^{\varepsilon}$ solves

$$
-\Delta v_{i}^{\varepsilon}=0 \text { in } B_{i}^{\varepsilon}, \quad v_{i}^{\varepsilon}=\mathcal{R}^{\varepsilon} \text { in } \partial B_{i}^{\varepsilon} .
$$

At this point we need to investigate the equation satisfied by $\mathcal{R}^{\varepsilon}$ to continue the asymptotic expansion. By subtracting (3.2) and (3.5), we obtain:

$$
-\Delta \mathcal{R}^{\varepsilon}=0 \text { in } \Omega^{\varepsilon}, \quad q \partial_{n} \mathcal{R}^{\varepsilon}=0 \text { on } \Sigma, \quad q \partial_{n} \mathcal{R}^{\varepsilon}=q_{i} \partial_{n} u_{i}^{\varepsilon}-q \partial_{n} u^{0} \text { on } \partial B_{i}^{\varepsilon} .
$$


By using (3.7) and (3.8) in the last equation of (3.10), we have on $\partial B_{i}^{\varepsilon}$

$$
\begin{aligned}
q \partial_{n} \mathcal{R}^{\varepsilon}= & q_{i} \nabla u^{0}\left(x_{i}\right) \cdot n-\varepsilon q_{i} D^{2} u^{0}\left(x_{i}\right)(n)^{2}+\frac{\varepsilon^{2}}{2} q_{i} D^{3} u^{0}\left(x_{i}\right)(n)^{3} \\
& +q_{i} \partial_{n} \mathcal{S}_{i}^{u}-q \partial_{n} u^{0}+q_{i} \partial_{n} v_{i}^{\varepsilon}
\end{aligned}
$$

where we have used the fact that $\left.\left(x-x_{i}\right)\right|_{\partial B_{i}^{\varepsilon}}=-\varepsilon n$. Note that in view of the boundary condition in (3.9), $\partial_{n} v_{i}^{\varepsilon}$ in (3.11) actually depends on $\mathcal{R}^{\varepsilon}$. The appropriate way to handle this is to introduce the Steklov-Poincaré operator or Dirichlet-to-Neumann operator $T_{i}$. Let $z \in H^{1}\left(\Omega^{\varepsilon}\right)$, then $T_{i}: H^{\frac{1}{2}}\left(\partial B_{i}^{\varepsilon}\right) \rightarrow H^{-\frac{1}{2}}\left(\partial B_{i}^{\varepsilon}\right)$ is defined as $T_{i}\left(\left.z\right|_{\partial B_{i}^{\varepsilon}}\right)=\left.\left(\partial_{n} \widehat{z}\right)\right|_{\partial B_{i}^{\varepsilon}}$, where $\widehat{z}$ is the solution of

$$
-\Delta \widehat{z}=0 \text { in } B_{i}^{\varepsilon}, \quad \widehat{z}=z \text { on } \partial B_{i}^{\varepsilon} .
$$

Therefore, $\left.\partial_{n} v_{i}^{\varepsilon}\right|_{\partial B_{i}^{\varepsilon}}=T_{i}\left(\mathcal{R}^{\varepsilon}\right)$, and equation (3.11) becomes

$q \partial_{n} \mathcal{R}^{\varepsilon}-q_{i} T_{i}\left(\mathcal{R}^{\varepsilon}\right)=\left(q_{i}-q\right)\left[\nabla u^{0}\left(x_{i}\right) \cdot n-\varepsilon D^{2} u^{0}\left(x_{i}\right)(n)^{2}+\frac{\varepsilon^{2}}{2} D^{3} u^{0}\left(x_{i}\right)(n)^{3}+\partial_{n} \mathcal{S}_{i}^{u}\right]$.

Note that $\partial_{n}(\cdot)$ denotes the operator $\nabla_{x}(\cdot) \cdot n$, which is easily related to the gradient with respect to $y_{i}$ by $\varepsilon \nabla_{x}(\cdot)=\nabla_{y_{i}}(\cdot)$.

To approximate $\mathcal{R}^{\varepsilon}$ we introduce the boundary layers $U_{i}, V_{i}, W_{i}$ and $\widetilde{U}_{i}^{j}$ described in section 3.1. The term $U_{i}$ is defined as the solution of

$$
\begin{aligned}
-\Delta_{y_{i}} U_{i} & =0 \quad \text { in } \quad \mathbb{R}^{N} \backslash \overline{B^{1}}, \quad U_{i} \rightarrow 0 \quad \text { at } \quad \infty, \\
q \partial_{n} U_{i}-q_{i} T_{i}\left(U_{i}\right) & =\left(q_{i}-q\right) \nabla u^{0}\left(x_{i}\right) \cdot n \quad \text { on } \partial B^{1} .
\end{aligned}
$$

Thus, $U_{i}$ is given by

$$
U_{i}\left(\varepsilon^{-1}\left(x-x_{i}\right)\right)=\frac{\alpha_{i}}{N-1} \frac{\varepsilon^{N}}{\left\|x-x_{i}\right\|^{N}} \nabla u^{0}\left(x_{i}\right) \cdot\left(x-x_{i}\right),
$$

with $\alpha_{i}$ defined as $\alpha_{i}=\left(q-q_{i}\right) /\left(q+\frac{q_{i}}{N-1}\right)$. The term $V_{i}$ is defined as the solution of (3.13) with the boundary condition

$$
q \partial_{n} V_{i}-q_{i} T_{i}\left(V_{i}\right)=-\varepsilon\left(q_{i}-q\right) D^{2} u^{0}\left(x_{i}\right)(n)^{2} \quad \text { on } \quad \partial B^{1} .
$$

Therefore, $V_{i}$ is given by

$$
V_{i}\left(\varepsilon^{-1}\left(x-x_{i}\right)\right)=\frac{\beta_{i}}{N} \frac{\varepsilon^{N+2}}{\left\|x-x_{i}\right\|^{N+2}} D^{2} u^{0}\left(x_{i}\right)\left(x-x_{i}\right)^{2},
$$

with $\beta_{i}$ defined as $\beta_{i}=\left(q-q_{i}\right)\left(q+\frac{2 q_{i}}{N}\right)^{-1}$. The term $W_{i}$ is defined as the solution of (3.13) with the boundary condition

$$
q \partial_{n} W_{i}-q_{i} T_{i}\left(W_{i}\right)=\frac{\varepsilon^{2}}{2}\left(q_{i}-q\right) D^{3} u^{0}\left(x_{i}\right)(n)^{3} \quad \text { on } \quad \partial B^{1} .
$$

Therefore, $W_{i}$ is given by

$$
W_{i}\left(\varepsilon^{-1}\left(x-x_{i}\right)\right)=c_{i} \frac{\varepsilon^{N+4}}{\left\|x-x_{i}\right\|^{N+4}} D^{3} u^{0}\left(x_{i}\right)\left(x-x_{i}\right)^{3},
$$


where $c_{i}$ is a constant which does not depend on $\varepsilon$. We do not need to give an explicit formulation for $c_{i}$ since it will not appear in the formula (3.55)-(3.58) of the topological derivative. In addition, the term $\widetilde{U}_{i}^{j}$ is the solution of (3.13) with the boundary condition

$$
q \partial_{n} \widetilde{U}_{i}^{j}-q_{i} T_{i}\left(\widetilde{U}_{i}^{j}\right)=-\left(q \partial_{n} U_{j}-q_{i} T_{i}\left(U_{j}\right)\right) \quad \text { on } \partial B^{1} .
$$

Note that, according to the definition of $T_{i}$ and $U_{j}$ we have $T_{i}\left(U_{j}\right)=\left.\partial_{n} U_{j}\right|_{\partial B_{j}^{\varepsilon}}$, and thus (3.20) becomes $q \partial_{n} \widetilde{U}_{i}^{j}-q_{i} T_{i}\left(\widetilde{U}_{i}^{j}\right)=\left(q_{i}-q\right) \partial_{n} U_{j}$ on $\partial B^{1}$. Let us calculate the gradient of $U_{j}$, namely

$$
\nabla_{x} U_{j}\left(\varepsilon^{-1}\left(x-x_{j}\right)\right)=\frac{\alpha_{j}}{N-1} \frac{\varepsilon^{N}}{\left\|x-x_{j}\right\|^{N}}\left(I-\frac{N}{\left\|x-x_{j}\right\|^{2}}\left(x-x_{j}\right) \otimes\left(x-x_{j}\right)\right) \nabla u^{0}\left(x_{j}\right) .
$$

On $\partial B_{i}^{\varepsilon}$ we set $x=x_{i}+\varepsilon z_{i}$, and in the above we observe that for all $x \in \partial B_{i}^{\varepsilon}$ we have

$$
\nabla_{x} U_{j}\left(\varepsilon^{-1}\left(x-x_{j}\right)\right)=\varepsilon^{N} A_{i j} \nabla u^{0}\left(x_{j}\right)+O\left(\varepsilon^{N+1}\right),
$$

where the matrix $A_{i j}$ is given by

$$
A_{i j}=\frac{\alpha_{j}}{N-1} \frac{1}{\left\|x_{i}-x_{j}\right\|^{N}}\left(I-\frac{N}{\left\|x_{i}-x_{j}\right\|^{2}}\left(x_{i}-x_{j}\right) \otimes\left(x_{i}-x_{j}\right)\right) .
$$

Thus, $\widetilde{U}_{i}^{j}$ is given by

$$
\widetilde{U}_{i}^{j}\left(\varepsilon^{-1}\left(x-x_{i}\right)\right)=\frac{\alpha_{i}}{N-1} \frac{\varepsilon^{2 N}}{\left\|x-x_{i}\right\|^{N}} A_{i j} \nabla u^{0}\left(x_{j}\right) \cdot\left(x-x_{i}\right)+O\left(\varepsilon^{2 N+1}\right) .
$$

The boundary layers $U_{i}, V_{i}, W_{i}$ and $\widetilde{U}_{i}^{j}$ do not satisfy the second equation in (3.10). Consequently, they leave a discrepancy on $\Sigma$ which will be compensated with the help of the function $g$ in (3.4) defined in the following way:

$$
-\Delta g=0 \text { in } \Omega, \quad q \partial_{n} g=-q \partial_{n} \sum_{i=1}^{n_{q}}\left(U_{i}+V_{i}+W_{i}+\sum_{j \neq i}^{n_{q}} \widetilde{U}_{i}^{j}\right) \text { on } \Sigma,
$$

It can be checked for the Neumann problem (3.25) that the compatibility condition is fulfilled.

REMARK 1. The coefficients of $A_{i j}$ given by (3.23) tend to infinity as $\left\|x_{i}-x_{j}\right\| \rightarrow 0$. Indeed, in the case of several inclusions, i.e. $n_{q}>1$, the asymptotic expansion (3.4) is valid only for inclusions which are not infinitesimally close to each other. Such a situation would require a different analysis, like the one in [3]. Thus, in numerical applications, situations where inclusions are close to each other have to be handled carefully in order to prevent wrong estimates due to the poor quality of the asymptotic expansion in such a situation.

REMARK 2. Although this is not apparent in the notation, (3.25) depends on $\left\{x_{i}\right\}_{i=1}^{n_{q}}$ through $U_{i}, V_{i}, W_{i}$ and $\widetilde{U}_{i}^{j}$. Thus, computationally $g$ is expensive to obtain since (3.25) has to be solved for every desirable configuration $\left\{x_{i}\right\}_{i=1}^{n_{q}}$. As a remedy, we introduce a function $h$ which alleviates the computational load. It depends only on the geometry of $\Omega$ and $\left\{x_{i}\right\}_{i=1}^{n_{q}}$ and can be pre-computed and stored, for fixed $\Omega$ and a set of possible configurations $\left\{x_{i}\right\}_{i=1}^{n_{q}}$.

For the subsequent analysis, we introduce a vector function $h(x, \xi)$, closely related to the gradient of the Green's function as the solution of the boundary value problem

$$
-\Delta_{x} h(x, \xi)=0 \text { in } \Omega, \quad q \partial_{n} h(x, \xi)=q \partial_{n} \frac{x-\xi}{\|x-\xi\|^{N}} \text { on } \Sigma .
$$


Note that the compatibility condition for the Neumann problem (3.26) is satisfied. We also impose the normalization condition $\int_{\Sigma} h(x, \xi) d x=0$ to obtain uniqueness. This allows to decompose $g$ according to

$$
g(x)=-\sum_{i=1}^{n_{q}} \frac{\alpha_{i}}{N-1} \varepsilon^{N} \nabla u^{0}\left(x_{i}\right) \cdot h\left(x, x_{i}\right)+O\left(\varepsilon^{N+2}\right) .
$$

Concerning $g$, we are now in a similar situation as we were for $u^{0}$, i.e., $g$ does not satisfy the boundary conditions on the boundaries of the holes $\partial B_{i}^{\varepsilon}$. Therefore, we repeat the above procedure for $g$. Namely, using a Taylor expansion around $x_{i}$ we get

$$
g(x)=g\left(x_{i}\right)+\nabla g\left(x_{i}\right) \cdot\left(x-x_{i}\right)+\mathcal{S}_{i}^{g}(x),
$$

where the remainder $\mathcal{S}_{i}^{g}$ is such that $\mathcal{S}_{i}^{g}(x)=2^{-1} D^{2} g\left(\zeta_{i}\right)\left(x-x_{i}\right)^{2}$ with $\zeta_{i} \in\left[x, x_{i}\right]$. Thus, we define the term $G_{i}$ in (3.4) as the solution of (3.13) and the boundary conditions

$$
q \partial_{n} G_{i}-q_{i} T_{i}\left(G_{i}\right)=\left(q_{i}-q\right) \nabla g\left(x_{i}\right) \cdot n \quad \text { on } \quad \partial B^{1} .
$$

Therefore, $G_{i}$ can be written as

$$
G_{i}\left(\varepsilon^{-1}\left(x-x_{i}\right)\right)=\frac{\alpha_{i}}{N-1} \frac{\varepsilon^{N}}{\left\|x-x_{i}\right\|^{N}} \nabla g\left(x_{i}\right) \cdot\left(x-x_{i}\right),
$$

To obtain (3.29) we have computed $T_{i}(g)$ using (3.12), which implies $T_{i}(g)=\nabla g\left(x_{i}\right) \cdot n+$ $\partial_{n} \mathcal{S}_{i}^{g}$. Finally, the remainder term $v^{\varepsilon}$ in the expansion (3.4) satisfies

$$
\begin{aligned}
-\Delta v^{\varepsilon} & =0 \quad \text { in } \quad \Omega^{\varepsilon}, \quad q \partial_{n} v^{\varepsilon}=-q \partial_{n} \sum_{i=1}^{n_{q}} G_{k} \quad \text { on } \quad \Sigma \\
q \partial_{n} v^{\varepsilon}-q_{i} T_{i}\left(v^{\varepsilon}\right) & =\left(q_{i}-q\right)\left(\frac{\varepsilon^{3}}{3 !} D^{4} u^{0}\left(\xi_{i}\right)(n)^{4}-\varepsilon D^{2} g\left(\zeta_{i}\right)(n)^{2}\right) \\
& +\left[-q \partial_{n}+q_{i} T_{i}\right]\left(\sum_{k \neq i}\left(V_{k}+W_{k}+\sum_{l \neq k} \widetilde{U}_{k}^{l}\right)+\sum_{k \neq i} G_{k}\right) \quad \text { on } \partial B_{i}^{\varepsilon} .
\end{aligned}
$$

3.3. Uniqueness of $u^{\varepsilon}$. The solution of (3.31)-(3.32) is not unique, but $v^{\varepsilon}$ is actually uniquely defined by the normalization conditions on $u^{0}$ and $u^{\varepsilon}$. Therefore, we decompose $v^{\varepsilon}$ in two parts $v^{\varepsilon}=\hat{v}^{\varepsilon}+\lambda^{\varepsilon}$, where $\lambda^{\varepsilon}$ is a constant that has to be determined and $\hat{v}^{\varepsilon}$ is the unique solution of (3.31)-(3.32) which satisfies the normalisation condition (2.4). Taking the integral over $\Sigma$ on both sides of (3.4) and using the decomposition of $v^{\varepsilon}$ we get

$$
\lambda^{\varepsilon}=-|\Sigma|^{-1} \int_{\Sigma} \sum_{i=1}^{n_{q}}\left(U_{i}+V_{i}+W_{i}+\sum_{j \neq i}^{n_{q}} \widetilde{U}_{i}^{j}\right)+g+\sum_{i=1}^{n_{q}} G_{i} .
$$

For our forthcoming expansion of the functional, we are only interested in the terms of order $O\left(\varepsilon^{N}\right)$ in $\lambda^{\varepsilon}$. Hence, we define $\lambda^{\varepsilon}=: \lambda_{1}^{\varepsilon}+\lambda_{2}^{\varepsilon}$

$$
\begin{aligned}
& \lambda_{1}^{\varepsilon}=-|\Sigma|^{-1} \int_{\Sigma} \sum_{i=1}^{n_{q}} U_{i}+g \\
& \lambda_{2}^{\varepsilon}=-|\Sigma|^{-1} \int_{\Sigma} \sum_{i=1}^{n_{q}}\left(V_{i}+W_{i}+\sum_{j \neq i}^{n_{q}} \widetilde{U}_{i}^{j}\right)+\sum_{i=1}^{n_{q}} G_{i} .
\end{aligned}
$$


3.4. Expansion of the tracking-type functional. As noted before, without the loss of generality we consider here the case of a single measurement, i.e. $M=1$. In addition, we assume that $\beta=0$ in order to neglect the perimeter term which is problematic for topological sensitivity. Indeed, the perimeter term in (2.5) contributes to the asymptotic expansion with a term $\mathcal{T}_{\mathcal{P}}(x) \varepsilon^{N-1}$, with $\mathcal{T}_{\mathcal{P}}(x)>0$ for all $x \in \Omega$, whereas the tracking-type integral contributes with a term $\mathcal{T}_{t r}(x) \varepsilon^{N}$. Consequently, for $\varepsilon$ small enough, the main term of the asymptotic expansion is only $\mathcal{T}_{\mathcal{P}}(x) \varepsilon^{N-1}$, always positive, and the topological derivative does not bring any useful information. We say that the perimeter is singular for the topological derivative, and for this reason we set $\beta=0$. Rather we capture the latter term by shape sensitivity in Section 4.1. In this respect, it is convenient to observe the order in $\varepsilon$ of each term of the expansion for $u^{\varepsilon}$, which are summarized in Table 3.1 below.

Invoking this assumption yields an expansion of the functional of order $\varepsilon^{2}$. Hence, the shape functional $J$ evaluated at $\Omega^{\varepsilon}$ is equal to

$$
J\left(\Omega^{\varepsilon}\right)=\int_{\Sigma}\left(u^{\varepsilon}-m\right)^{2} .
$$

Plugging (3.4) in (3.35) we get

$$
\begin{aligned}
J\left(\Omega^{\varepsilon}\right)= & J(\Omega)+\sum_{i=1}^{n_{q}}\left(K_{U_{i}}+K_{V_{i}}+K_{W_{i}}+\sum_{j \neq i}^{n_{q}} K_{\widetilde{U}_{i}^{j}}\right) \\
& +K_{g}+\sum_{i=1}^{n_{q}} K_{G_{i}}+K_{v^{\varepsilon}}+K_{\lambda^{\varepsilon}}+L,
\end{aligned}
$$

where $K_{\phi}$ denotes the integral $K_{\phi}=\int_{\Sigma} 2 \phi(x)\left(u^{0}(x)-m(x)\right) d x$ for any function $\phi$ and

$$
L=\int_{\Sigma}\left(\sum_{i=1}^{n_{q}}\left(U_{i}+V_{i}+W_{i}+\sum_{j \neq i}^{n_{q}} \widetilde{U}_{i}^{j}\right)+g+\sum_{i=1}^{n_{q}} G_{i}+v^{\varepsilon}\right)^{2}
$$

Now we analyse each term in (3.36) separately. We start by noting that in view of (2.3)-(2.4) we have $K_{\lambda^{\varepsilon}}=0$. Next we introduce the adjoint state $p$ as the solution of

$$
-\Delta p=0 \text { in } \Omega, \quad \partial_{n} p=2\left(u^{0}-m\right) \text { on } \Sigma .
$$

Note that the compatibility condition for $p$ is obviously satisfied.

TABLE 3.1

Orders of $\varepsilon$ for the terms of expansion (3.4). The orders between parenthesis correspond to the behaviour on $\partial B_{i}^{\varepsilon}$, while the orders without parenthesis correspond to the behaviour "far" from the inclusions $B_{i}^{\varepsilon}$.

\begin{tabular}{cccccccc}
\hline & $U_{i}$ & $V_{i}$ & $W_{i}$ & $\widetilde{U}_{i}^{j}$ & $g$ & $G_{i}$ & $\lambda^{\varepsilon}$ \\
\hline$N$ & $\varepsilon^{N}\left(\varepsilon^{1}\right)$ & $\varepsilon^{N+2}\left(\varepsilon^{2}\right)$ & $\varepsilon^{N+4}\left(\varepsilon^{3}\right)$ & $\varepsilon^{2 N}\left(\varepsilon^{N+1}\right)$ & $\varepsilon^{N}$ & $\varepsilon^{2 N}\left(\varepsilon^{N+1}\right)$ & $\varepsilon^{N}$ \\
\hline$N=2$ & $\varepsilon^{2}\left(\varepsilon^{1}\right)$ & $\varepsilon^{4}\left(\varepsilon^{2}\right)$ & $\varepsilon^{6}\left(\varepsilon^{3}\right)$ & $\varepsilon^{4}\left(\varepsilon^{3}\right)$ & $\varepsilon^{2}$ & $\varepsilon^{4}\left(\varepsilon^{3}\right)$ & $\varepsilon^{2}$ \\
\hline$N=3$ & $\varepsilon^{3}\left(\varepsilon^{1}\right)$ & $\varepsilon^{5}\left(\varepsilon^{2}\right)$ & $\varepsilon^{7}\left(\varepsilon^{3}\right)$ & $\varepsilon^{6}\left(\varepsilon^{4}\right)$ & $\varepsilon^{3}$ & $\varepsilon^{6}\left(\varepsilon^{4}\right)$ & $\varepsilon^{3}$ \\
\hline
\end{tabular}


3.4.1. Calculation of $K_{U_{i}}$. The main tool to proceed the terms $K_{\phi}$ is Green's formula and the use of the adjoint state $p$. We transform the integrals on $\Sigma$ to integrals on $\Gamma_{i}^{\varepsilon}:=\partial B_{i}^{\varepsilon}$ and in $\Omega^{\varepsilon}$. The integrals on $\Omega^{\varepsilon}$ always vanish due to the harmonic nature of the functions under consideration. The boundary integrals on $\Gamma_{i}^{\varepsilon}$ can be computed explicitely using Taylor expansions. For the sake of compactness, we do not provide too many details on the calculation here. Rather we defer complete calculations to the appendix. We start with a general computation of $K_{\phi}$ for some harmonic function. Using Green's formula, we find

$$
\int_{\Omega^{\varepsilon}}-\Delta p(x) \phi\left(y_{i}\right)+p(x) \Delta \phi\left(y_{i}\right) d x=\int_{\Sigma \cup \cup_{j} \Gamma_{j}^{\varepsilon}}-\partial_{n} p(x) \phi\left(y_{i}\right)+p(x) \partial_{n} \phi\left(y_{i}\right) d x=0,
$$

and, thus, get

$$
\begin{aligned}
K_{\phi}=\int_{\Sigma} \partial_{n} p(x) \phi\left(y_{i}\right) d x= & -\int_{\Gamma_{i}^{\varepsilon}} \partial_{n} p(x) \phi\left(y_{i}\right) d x+\int_{\Gamma_{i}^{\varepsilon}} p(x) \partial_{n} \phi\left(y_{i}\right) d x \\
& +\int_{\Sigma} p(x) \partial_{n} \phi\left(y_{i}\right) d x-\sum_{j \neq i} \int_{\Gamma_{j}^{\varepsilon}} \partial_{n} p(x) \phi\left(y_{i}\right)-p(x) \partial_{n} \phi\left(y_{i}\right) d x .
\end{aligned}
$$

Taking $\phi=U_{i}$, we readily check that

$$
\int_{\Gamma_{i}^{\varepsilon}} \partial_{n} p(x) U_{i}\left(y_{i}\right) d x=-\frac{1}{N-1} \int_{\Gamma_{i}^{\varepsilon}} p(x) \partial_{n} U_{i}\left(y_{i}\right) d x .
$$

Indeed, using an harmonic extension of $U_{i \mid \Gamma_{i}^{\varepsilon}}$ in $B_{i}^{\varepsilon}$, and Green's formula we obtain

$$
\int_{\Gamma_{i}^{\varepsilon}} \partial_{n} p(x) U_{i}\left(y_{i}\right) d x=\int_{\Gamma_{i}^{\varepsilon}} p(x) T_{i}\left(U_{i}\right)\left(y_{i}\right) d x .
$$

Recall that we have already computed $\nabla_{x} U_{i}\left(y_{i}\right)$ in (3.21). Now, since $x-x_{i}=-\varepsilon n$ on $\Gamma_{i}^{\varepsilon}$ we have $\partial_{n} U_{i}\left(y_{i}\right)_{\mid \Gamma_{i}^{\varepsilon}}=-\alpha_{i} \nabla_{x} u^{0}\left(x_{i}\right) \cdot n=-(N-1) T_{i}\left(U_{i}\right)$, which yields (3.40). Using a third-order Taylor expansion of $p$ at $x=x_{i}$ we compute

$$
\begin{aligned}
& \int_{\Gamma_{i}^{\varepsilon}} p(x) \partial_{n} U_{i}\left(y_{i}\right) d x=-\alpha_{i} \int_{\Gamma_{i}^{\varepsilon}} p\left(x_{i}\right) \nabla_{x} u^{0}\left(x_{i}\right) \cdot n d x+\varepsilon \alpha_{i} \int_{\Gamma_{i}^{\varepsilon}} \nabla p\left(x_{i}\right) \cdot n \nabla_{x} u^{0}\left(x_{i}\right) \cdot n d x \\
& -\frac{\varepsilon^{2}}{2} \alpha_{i} \int_{\Gamma_{\varepsilon}} D^{2} p\left(x_{i}\right)(n)^{2} \nabla_{x} u^{0}\left(x_{i}\right) \cdot n d x+\frac{\varepsilon^{3}}{3 !} \alpha_{i} \int_{\Gamma_{i}^{\varepsilon}} D^{3} p\left(x_{i}\right)(n)^{3} \nabla_{x} u^{0}\left(x_{i}\right) \cdot n d x+O\left(\varepsilon^{N+3}\right) \\
= & \alpha_{i}\left|B_{i}^{\varepsilon}\right| \nabla_{x} u^{0}\left(x_{i}\right) \cdot \nabla p\left(x_{i}\right)+\frac{\alpha_{i}}{2(N+2)} \varepsilon^{2}\left|B_{i}^{\varepsilon}\right| \nabla\left(\Delta p\left(x_{i}\right)\right) \cdot \nabla u^{0}\left(x_{i}\right)+O\left(\varepsilon^{N+3}\right) .
\end{aligned}
$$

Note that we have $\left|B_{i}^{\varepsilon}\right|=\pi \varepsilon^{2}$ for $N=2$ and $\left|B_{i}^{\varepsilon}\right|=\frac{4}{3} \pi \varepsilon^{3}$ for $N=3$. As the function $p$ is harmonic and in view of (3.40) we finally obtain

$$
\begin{aligned}
K_{U_{i}} & =\frac{N}{N-1} \alpha_{i}\left|B_{i}^{\varepsilon}\right| \nabla_{x} u^{0}\left(x_{i}\right) \cdot \nabla p\left(x_{i}\right)+O\left(\varepsilon^{N+3}\right) \\
& +\int_{\Sigma} p(x) \partial_{n} U_{i}\left(y_{i}\right) d x+\sum_{j \neq i} \int_{\Gamma_{j}^{\varepsilon}} \partial_{n} p(x) U_{i}\left(y_{i}\right)+p(x) \partial_{n} U_{i}\left(y_{i}\right) d x .
\end{aligned}
$$

Below we shall see that the first integral in (3.42) will be canceled by a term coming from $K_{g}$. The integrals in the sum in (3.42) vanish according to Green's formula (3.38). 
3.4.2. Calculation of $K_{V_{i}}$. We take $\phi=V_{i}$ in (3.39). As in (3.40) we have

$$
\int_{\Gamma_{i}^{\varepsilon}} \partial_{n} p(x) V_{i}\left(y_{i}\right) d x=-\frac{2}{N} \int_{\Gamma_{i}^{\varepsilon}} p(x) \partial_{n} V_{i}\left(y_{i}\right) d x .
$$

Using a Taylor expansion for $\partial_{n} p(x)$ about $x_{i}$ we get for the main integral

$$
\begin{aligned}
-\int_{\Gamma_{i}^{\varepsilon}} \partial_{n} p(x) V_{i}\left(y_{i}\right) d x & =-\int_{\Gamma_{i}^{\varepsilon}} \nabla p\left(x_{i}\right) \cdot n V_{i}\left(y_{i}\right) d x+\varepsilon \int_{\Gamma_{i}^{\varepsilon}} D^{2} p\left(x_{i}\right)(n)^{2} V_{i}\left(y_{i}\right) d x+O\left(\varepsilon^{N+3}\right) \\
& =\varepsilon \int_{\Gamma_{i}^{\varepsilon}} D^{2} p\left(x_{i}\right)(n)^{2} V_{i}\left(y_{i}\right) d x+O\left(\varepsilon^{N+3}\right) \\
& =\frac{\beta_{i}}{N} \varepsilon^{3} \int_{\Gamma_{i}^{\varepsilon}} D^{2} p\left(x_{i}\right)(n)^{2} D^{2} u^{0}\left(x_{i}\right)(n)^{2} d x+O\left(\varepsilon^{N+3}\right) \\
& =\frac{\beta_{i}}{N(N+2)} \varepsilon^{2}\left|B_{i}^{\varepsilon}\right|\left(2 D^{2} p\left(x_{i}\right) \cdot D^{2} u^{0}\left(x_{i}\right)+\Delta p\left(x_{i}\right) \Delta u^{0}\left(x_{i}\right)\right)+O\left(\varepsilon^{N+3}\right)
\end{aligned}
$$

Since $u^{0}$ and $p$ are harmonic functions and using (3.43) we obtain

$$
\begin{aligned}
K_{V_{i}} & =\frac{\beta_{i}}{N} \varepsilon^{2}\left|B_{i}^{\varepsilon}\right| D^{2} p\left(x_{i}\right) \cdot D^{2} u^{0}\left(x_{i}\right)+O\left(\varepsilon^{N+3}\right) \\
& +\int_{\Sigma} p(x) \partial_{n} V_{i}\left(y_{i}\right) d x+\sum_{j \neq i} \int_{\Gamma_{j}^{\varepsilon}} \partial_{n} p(x) V_{i}\left(y_{i}\right)+p(x) \partial_{n} V_{i}\left(y_{i}\right) d x .
\end{aligned}
$$

As before, we shall see below that the first integral in (3.45) will be canceled by a term coming from $K_{g}$. The integrals in the sum in (3.45) vanish according to Green's formula (3.38).

3.4.3. Calculation of $K_{W_{i}}$. We take $\phi=W_{i}$ in (3.39). The integral on $\Sigma$ will be canceled by a term coming from $K_{g}$, and the integrals on $\Gamma_{j}^{\varepsilon}$ for $j \neq i$ vanish as usual. The integrals on $\Gamma_{i}^{\varepsilon}$ are of order $O\left(\varepsilon^{N+3}\right)$. Indeed, the main term vanishes since $u^{0}$ is harmonic and

$$
\varepsilon^{3} \int_{\partial B_{i}^{\varepsilon}} D^{3} u^{0}\left(x_{i}\right)(n)^{3} \nabla p\left(x_{i}\right) \cdot n=\frac{3}{N+2} \varepsilon^{2}\left|B_{i}^{\varepsilon}\right| \nabla\left(\Delta u^{0}\left(x_{i}\right)\right) \cdot \nabla p\left(x_{i}\right)=0 .
$$

3.4.4. Calculation of $K_{\widetilde{U}_{i}^{j}}$. Taking $\phi=\widetilde{U}_{i}^{j}$ and splitting the sum over $k$, we get

$$
\begin{aligned}
\sum_{j \neq i} K_{\widetilde{U}_{i}^{j}}= & -\sum_{j \neq i} \int_{\Sigma} \partial_{n} p(x) \widetilde{U}_{i}^{j}\left(y_{i}\right) d x-\int_{\Sigma} p(x) \partial_{n} \widetilde{U}_{i}^{j}\left(y_{i}\right) d x \\
& -\sum_{j \neq i} \int_{\Gamma_{i}^{\varepsilon}} \partial_{n} p(x) \widetilde{U}_{i}^{j}\left(y_{i}\right)-p(x) \partial_{n} \widetilde{U}_{i}^{j}\left(y_{i}\right) d x \\
& -\sum_{j \neq i} \sum_{k \neq i} \int_{\Gamma_{k}^{\varepsilon}} \partial_{n} p(x) \widetilde{U}_{i}^{j}\left(y_{i}\right)-p(x) \partial_{n} \widetilde{U}_{i}^{j}\left(y_{i}\right) d x
\end{aligned}
$$

The sum of integrals (3.48) vanishes according to Green's formula. The term (3.47) is computed as in (3.41), and we obtain

$$
\begin{aligned}
\sum_{j \neq i} K_{\widetilde{U}_{i}^{j}}= & -\sum_{j \neq i} \int_{\Sigma} \partial_{n} p(x) \widetilde{U}_{i}^{j}\left(y_{i}\right) d x-\int_{\Sigma} p(x) \partial_{n} \widetilde{U}_{i}^{j}\left(y_{i}\right) d x \\
& +\frac{N}{N-1} \alpha_{i} \varepsilon^{N}\left|B_{i}^{\varepsilon}\right| A_{i j} \nabla_{x} u^{0}\left(x_{j}\right) \cdot \nabla p\left(x_{i}\right)+O\left(\varepsilon^{2 N+3}\right) .
\end{aligned}
$$

The sum of integrals in (3.49) will be canceled by a term in $K_{g}$. 
3.4.5. Calculation of $K_{g}$. Since $p$ and $g$ are both harmonic in $\Omega$ we have $K_{g}=\int_{\Sigma} p(x) \partial_{n} g(x) d x$. According to the boundary condition in (3.25), $K_{g}$ cancels out the sum of integrals of (3.42), (3.45) and (3.46).

3.4.6. Calculation of $K_{G_{i}}$. In a similar way as for $K_{U_{i}}$ we get

$$
K_{G_{i}}=\frac{N}{N-1}\left|B_{i}^{\varepsilon}\right| \alpha_{i} \nabla_{x} g\left(x_{i}\right) \cdot \nabla p\left(x_{i}\right)+O\left(\varepsilon^{2 N+3}\right)+\int_{\Sigma} p(x) \partial_{n} G_{i}\left(y_{i}\right) d x
$$

The integral in (3.51) will be canceled by a term from $K_{v^{\varepsilon}}$. The main term in (3.51) can be further simplified using (3.27):

$$
\frac{N}{N-1}\left|B_{i}^{\varepsilon}\right| \alpha_{i} \nabla_{x} g\left(x_{i}\right) \cdot \nabla p\left(x_{i}\right)=-\sum_{j=1}^{n_{q}} \frac{N}{(N-1)^{2}}\left|B_{i}^{\varepsilon}\right| \varepsilon^{N} \alpha_{i} \alpha_{j} \nabla_{x} u^{0}\left(x_{j}\right) \cdot \nabla h\left(x_{i}, x_{j}\right) \cdot \nabla p\left(x_{i}\right) .
$$

Note that $\nabla h\left(x_{i}, x_{j}\right)$ in (3.52) is an $N \times N$ matrix.

3.4.7. Calculation of $K_{v^{\varepsilon}}$. Using Green's formula we get

$$
K_{v^{\varepsilon}}=\int_{\Sigma} p(x) \partial_{n} v^{\varepsilon}(x) d x-\sum_{k=1}^{n_{q}} \int_{\Gamma_{k}^{\varepsilon}} \partial_{n} p(x) v^{\varepsilon}(x)-p(x) \partial_{n} v^{\varepsilon}(x) d x .
$$

The first integral in (3.53) cancels with the integral in (3.51) due to the boundary condition in (3.31). The lowest-order terms on the right-hand side of (3.32) are of order $O\left(\varepsilon^{3}\right)$. Thus, the sum in (3.53) is of order $O\left(\varepsilon^{N+3}\right)$.

3.4.8. Calculation of $L$. In $L$, we only keep the terms of order $O\left(\varepsilon^{N}\right)$ inside the square, so that $L$ can be written as $L=L_{0}^{\varepsilon}+O\left(\varepsilon^{2 N+1}\right)$, with

$$
\begin{aligned}
L_{0}^{\varepsilon} & =\int_{\Sigma}\left(\sum_{i=1}^{n_{q}} U_{i}+g+\lambda_{1}^{\varepsilon}\right)^{2} d x \\
& =\sum_{k, l=1}^{n_{q}} \int_{\Sigma} U_{k} U_{l}+\int_{\Sigma} g^{2}+|\Sigma|\left(\lambda_{1}^{\varepsilon}\right)^{2}+2 \sum_{i=1}^{n_{q}} \int_{\Sigma} g U_{i}+2 \lambda_{1}^{\varepsilon} \int_{\Sigma} \sum_{i=1}^{n_{q}} U_{i}+g \\
& =\sum_{k, l=1}^{n_{q}} \int_{\Sigma} U_{k} U_{l}+\int_{\Sigma} g^{2}+2 \sum_{i=1}^{n_{q}} \int_{\Sigma} g U_{i}-|\Sigma|\left(\lambda_{1}^{\varepsilon}\right)^{2} .
\end{aligned}
$$

We further obtain

$$
\int_{\Sigma} U_{i}=\frac{\alpha_{i}}{N-1} \varepsilon^{N} \nabla u^{0}\left(x_{i}\right) \cdot I_{i}, \int_{\Sigma} U_{k} U_{l}=\frac{\alpha_{k} \alpha_{l}}{(N-1)^{2}} \varepsilon^{2 N}\left[\nabla u^{0}\left(x_{k}\right) \otimes \nabla u^{0}\left(x_{l}\right)\right] \cdot I_{k, l},
$$

with $I_{i}=\int_{\Sigma}\left(x-x_{i}\right)\left\|x-x_{i}\right\|^{-N}$ and $I_{k, l}=\int_{\Sigma} \frac{x-x_{k}}{\left\|x-x_{k}\right\|^{N}} \otimes \frac{x-x_{l}}{\left\|x-x_{l}\right\|^{N}}$. The integrals depending on $g$ simplify due to (3.27)

$$
\int_{\Sigma} g^{2}=\sum_{k, l=1}^{n_{q}} \frac{\alpha_{k} \alpha_{l}}{(N-1)^{2}} \varepsilon^{2 N}\left[\nabla u^{0}\left(x_{k}\right) \otimes \nabla u^{0}\left(x_{l}\right)\right] \cdot I_{k, l}^{h}+O\left(\varepsilon^{2 N+2}\right)
$$

with $I_{k, l}^{h}=\int_{\Sigma} h\left(x, x_{k}\right) \otimes h\left(x, x_{l}\right) d x$. We also have for the mixed term

$$
\sum_{i=1}^{n_{q}} \int_{\Sigma} g U_{i}=-\sum_{i, j=1}^{n_{q}} \frac{\alpha_{i} \alpha_{j}}{(N-1)^{2}} \varepsilon^{2 N}\left[\nabla u^{0}\left(x_{i}\right) \otimes \nabla u^{0}\left(x_{j}\right)\right] \cdot I_{i, j}^{m}+O\left(\varepsilon^{2 N+2}\right)
$$


with $I_{i, j}^{m}=\int_{\Sigma}\left\|x-x_{i}\right\|^{-N}\left(x-x_{i}\right) \otimes h\left(x, x_{j}\right)$. Note that $\lambda_{1}^{\varepsilon}$ is given by (3.33) and can be further simplified according to (3.27):

$$
\lambda_{1}^{\varepsilon}=-|\Sigma|^{-1} \sum_{i=1}^{n_{q}} \frac{\alpha_{i}}{(N-1)} \varepsilon^{N} \nabla u^{0}\left(x_{i}\right) \cdot I_{i}+O\left(\varepsilon^{N+2}\right) .
$$

REMARK 3. The integrals $I_{i}, I_{k, l}, I_{k, l}^{h}$ and $I_{i, j}^{m}$ in (3.54) depend only on the geometry of $\Omega$. They can be computed at the beginning of the optimization process and stored for further computations. The computation of $I_{i}$ and $I_{k, l}$ might even be explicit if the geometry of $\Omega$ is simple, e.g. in the case of a rectangle. On the other hand, the computation of $I_{i}^{h}, I_{k, l}^{h}$ and $I_{i, j}^{m}$ requires to solve (3.26) at every point of the domain, which costly on fine meshes. Approximation by interpolation from a coarse grid reduces the computational load.

3.4.9. Expansion of the functional. Gathering the previous results for the expansion of (3.35) we obtain

$J\left(\Omega^{\varepsilon}\right)=J(\Omega)+\sum_{i=1}^{n_{q}}\left(\mathcal{T}_{0, \varepsilon}^{i}\left(x_{i}\right)+\mathcal{T}_{1, \varepsilon}^{i}\left(x_{i}\right)+\sum_{j=1}^{n_{q}} \mathcal{T}_{2, \varepsilon}^{i, j}\left(x_{i}, x_{j}\right)+\sum_{j \neq i} \mathcal{T}_{3, \varepsilon}^{i, j}\left(x_{i}, x_{j}\right)\right)+L_{0}^{\varepsilon}+O\left(\varepsilon^{N+3}\right)$,

with

$$
\begin{aligned}
\mathcal{T}_{0, \varepsilon}^{i}\left(x_{i}\right) & =\frac{N}{N-1} \alpha_{i}\left|B_{i}^{\varepsilon}\right| \nabla u^{0}\left(x_{i}\right) \cdot \nabla p\left(x_{i}\right), \\
\mathcal{T}_{1, \varepsilon}^{i}\left(x_{i}\right) & =\frac{\beta_{i}}{N} \varepsilon^{2}\left|B_{i}^{\varepsilon}\right| D^{2} u^{0}\left(x_{i}\right) \cdot D^{2} p\left(x_{i}\right), \\
\mathcal{T}_{2, \varepsilon}^{i, j}\left(x_{i}, x_{j}\right) & =-\frac{N}{(N-1)^{2}}\left|B_{i}^{\varepsilon}\right| \varepsilon^{N} \alpha_{i} \alpha_{j} \nabla u^{0}\left(x_{j}\right) \cdot \nabla h\left(x_{i}, x_{j}\right) \cdot \nabla p\left(x_{i}\right), \\
\mathcal{T}_{3, \varepsilon}^{i, j}\left(x_{i}, x_{j}\right) & =\frac{N}{N-1} \alpha_{i} \varepsilon^{N}\left|B_{i}^{\varepsilon}\right| A_{i j} \nabla u^{0}\left(x_{j}\right) \cdot \nabla p\left(x_{i}\right),
\end{aligned}
$$

and $L_{0}^{\varepsilon}=L_{0}^{\varepsilon}\left(\left\{x_{i}\right\}_{i=1}^{n_{q}}\right)$ given by (3.54).

REMARK 4. Note that the orders of the quantities $\mathcal{T}_{k, \varepsilon}^{i}, k=0,1,2,3$ are $\mathcal{T}_{0, \varepsilon}^{i}\left(x_{i}\right)=$ $O\left(\varepsilon^{N}\right), \mathcal{T}_{1, \varepsilon}^{i}\left(x_{i}\right)=O\left(\varepsilon^{N+2}\right), \mathcal{T}_{2, \varepsilon}^{i}\left(x_{i}\right)=O\left(\varepsilon^{2 N}\right)$, and $\mathcal{T}_{3, \varepsilon}^{i, j}\left(x_{i}, x_{j}\right)=O\left(\varepsilon^{2 N}\right)$. Since $2 N=N+3$ for $N=3, \mathcal{T}_{2, \varepsilon}^{i, j}\left(x_{i}\right), \mathcal{T}_{3, \varepsilon}^{i, j}\left(x_{i}\right)$ and $L_{0}^{\varepsilon}$ are of the same order as the rest in (3.55) and should not, therefore, be taken into account for numerical tests when $N=3$.

REMARK 5. The terms $\mathcal{T}_{3, \varepsilon}^{i, j}\left(x_{i}, x_{j}\right)$ appear in (3.55) only if $n_{q}>1$, i.e. if several objects with different conductivities are to be reconstructed. They correspond to interaction between the inclusions. In the case $N=3$, even if $n_{q}>1, \mathcal{T}_{3, \varepsilon}^{i, j}\left(x_{i}, x_{j}\right)$ is negligible and is of the order $\varepsilon^{6}$ of the remainder. Note also that the term $\mathcal{T}_{2, \varepsilon}^{i, j}\left(x_{i}, x_{j}\right)$ is negligible in three dimensions.

4. Shape and conductivity derivatives. Our higher-order topological derivatives will be used to efficiently initialize the overall algorithm for detecting the inclusions in EIT. The non-local attributes of the higher-order terms in the topological derivative (3.55) (see Remark 2) make it difficult to use them in a method using these higher-order expansions iteratively. We thus rely on the notion of shape derivative [11,23] based on the velocity method to evolve the interface obtained using the topological derivative in the initialization phase. In section 4.2 , we study the sensitivity of the conductivities with respect to the scalar values $q_{i}$. 
4.1. Shape derivative of the functional. For shape optimization purposes, we consider a different framework than for the topological derivative. For this purpose, we introduce the domains $\Omega^{*}=\Omega \backslash\left(\cup_{i=1}^{n_{q}} \overline{\Omega_{i}}\right)$ with $\Omega_{i} \cap \Omega_{j}=\varnothing$ for $i \neq j$, and still use $\Sigma=\partial \Omega$ and $\Gamma_{i}=\partial \Omega_{i}$. The analogues of problems (3.2) and (3.3) in the domains $\Omega_{i}$ and $\Omega^{*}$ are

$$
\begin{aligned}
-\Delta u & =0 \text { in } \Omega^{*}, \quad q \partial_{n} u=f \text { on } \Sigma, \quad q \partial_{n} u=q_{i} \partial_{n} u_{i} \text { on } \Gamma_{i}, \\
-\Delta u_{i} & =0 \text { in } \Omega_{i}, \quad u_{i}=u \text { on } \Gamma_{i} .
\end{aligned}
$$

In the framework of the velocity method, we choose $V: \mathbb{R}^{N} \rightarrow \mathbb{R}^{N}$ to be a given smooth vector field with compact support in $\mathbb{R}^{N}$. According to [23], the shape derivatives $u_{i}^{\prime}$ and $u^{\prime}$ of $u_{i}$ and $u$, respectively, solve the following systems

$$
\begin{aligned}
& -\Delta u^{\prime}=0 \text { in } \Omega^{*}, \quad q \partial_{n} u^{\prime}=0 \text { on } \Sigma, \\
& q \partial_{n} u^{\prime}=q_{i} \partial_{n} u_{i}^{\prime}-\frac{\partial^{2}}{\partial n^{2}}\left(q u-q_{i} u_{i}\right) v_{n}+\nabla_{\Gamma}\left(q u-q_{i} u_{i}\right) \cdot \nabla_{\Gamma} v_{n} \text { on } \Gamma_{i},
\end{aligned}
$$

and further

$$
-\Delta u_{i}^{\prime}=0 \text { in } \Omega_{i}, \quad u_{i}^{\prime}=\partial_{n}\left(u-u_{i}\right) v_{n}+u^{\prime} \text { in } \Gamma_{i},
$$

where the tangential gradient $\nabla_{\Gamma}$ is defined as $\nabla_{\Gamma}(\cdot)=\nabla(\cdot)-\partial_{n}(\cdot) n$, and $v_{n}=V \cdot n$. Notice that in all equations, and in particular (4.4) and (4.5), $n$ denotes the outer normal vector to $\partial \Omega^{*}$. Therefore it is the inner normal vector to $\partial \Omega_{i}$. Note that this does not affect (4.5) since $n$ appears once on both sides of this equation. This consideration is also important for the calculation of the shape derivative in section 4.1, for instance when considering signs in Green's formulae.

The derivative of the functional in (2.5) with respect to the shape is

$$
d J\left(\left\{\Omega_{i}\right\}_{i=1}^{n_{q}} ; V\right)=\int_{\Sigma} 2(u-m) u^{\prime}+\beta \sum_{i=1}^{n_{q}}\left|q-q_{i}\right| \int_{\Gamma_{i}} \mathcal{H} v_{n},
$$

where $\mathcal{H}$ is the mean curvature of the boundary of $\Omega^{*}$. In order to further study the integral over $\Sigma$ in (4.6), we introduce the following adjoint states $p$ and $p_{i}$ as the solutions to

$$
\begin{aligned}
-\Delta p & =0 \text { in } \Omega^{*}, \quad q \partial_{n} p=2(u-m) \text { on } \Sigma, \quad q \partial_{n} p=q_{i} \partial_{n} p_{i} \text { on } \Gamma_{i}, \\
-\Delta p_{i} & =0 \text { in } \Omega_{i}, \quad p_{i}=p \text { on } \Gamma_{i},
\end{aligned}
$$

respectively. Applying Green's formula gives

$$
\begin{aligned}
0=\int_{\Omega}-u^{\prime} \Delta p+p \Delta u^{\prime} & =\sum_{i=1}^{n_{q}} \int_{\Gamma_{i}}-u^{\prime} \partial_{n} p+\partial_{n} u^{\prime} p+\int_{\Sigma}-u^{\prime} \partial_{n} p+\partial_{n} u^{\prime} p, \\
0=\int_{\Omega_{i}}-u_{i}^{\prime} \Delta p_{i}+p_{i} \Delta u_{i}^{\prime} & =-\int_{\Gamma_{i}}-u_{i}^{\prime} \partial_{n} p_{i}+p_{i} \partial_{n} u_{i}^{\prime} .
\end{aligned}
$$

In view of the boundary conditions in (4.7) and using (4.3),(4.9) and (4.4) we get

$$
\begin{aligned}
\int_{\Sigma} 2(u-m) q^{-1} u^{\prime}= & \sum_{i=1}^{n_{q}} \int_{\Gamma_{i}}-u^{\prime} \partial_{n} p+\partial_{n} u^{\prime} p+\int_{\Sigma} \partial_{n} u^{\prime} p \\
= & \sum_{i=1}^{n_{q}}\left(\int_{\Gamma_{i}}-u^{\prime} \partial_{n} p+p q^{-1} q_{i} \partial_{n} u_{i}^{\prime}\right. \\
& \left.+\int_{\Gamma_{i}}-p q^{-1} \frac{\partial^{2}}{\partial n^{2}}\left(q u-q_{i} u_{i}\right) v_{n}+\int_{\Gamma_{i}} p q^{-1} \nabla_{\Gamma}\left(q u-q_{i} u_{i}\right) \cdot \nabla_{\Gamma} v_{n}\right) .
\end{aligned}
$$


In view of (4.10) and (4.5) we have

$$
\int_{\Gamma_{i}} p q^{-1} q_{i} \partial_{n} u_{i}^{\prime}=\int_{\Gamma_{i}} u_{i}^{\prime} \partial_{n} p=\int_{\Gamma_{i}} \partial_{n} p_{i} \partial_{n}\left(u-u_{i}\right) v_{n}+u^{\prime} \partial_{n} p .
$$

Therefore we conclude

$$
\begin{aligned}
\int_{\Sigma} 2(u-m) q^{-1} u^{\prime}= & \sum_{i=1}^{n_{q}}\left(\int_{\Gamma_{i}} \partial_{n} p_{i} \partial_{n}\left(u-u_{i}\right) v_{n}\right. \\
& \left.+\int_{\Gamma_{i}}-p q^{-1} \frac{\partial^{2}}{\partial n^{2}}\left(q u-q_{i} u_{i}\right) v_{n}+\int_{\Gamma_{i}} p q^{-1} \nabla_{\Gamma}\left(q u-q_{i} u_{i}\right) \cdot \nabla_{\Gamma} v_{n}\right) .
\end{aligned}
$$

In order to further process the right hand side above, we rely on the following proposition; see [14, Prop. 5.4.12].

PROPOSITION 4.1. Let $\Omega$ be an open set of class $\mathcal{C}^{2}$ and $u: \bar{\Omega} \rightarrow \mathbb{R}$ of class $\mathcal{C}^{2}$. Let $n$ be the outer unit normal vector to $\Omega$ and $\mathcal{H}$ the curvature of $\Gamma=\partial \Omega$. Then $\Delta u=$ $\Delta_{\Gamma} u+\mathcal{H} \partial_{n} u+\partial_{n}^{2} u$ on $\Gamma$, where $\Delta_{\Gamma}$ is the so-called Laplace-Beltrami operator on $\Gamma$.

Since $\Delta u_{i}=\Delta u=0$ and $\partial_{n}\left(q u-q_{i} u_{i}\right)=0$ on $\Gamma$, applying Proposition 4.1 we have

$$
\begin{aligned}
& \int_{\Sigma} 2(u-m) q^{-1} u^{\prime} \\
= & \sum_{i=1}^{n_{q}} \int_{\Gamma_{i}} \partial_{n} p_{i} \partial_{n}\left(u-u_{i}\right) v_{n}+\int_{\Gamma_{i}} p q^{-1}\left[\nabla_{\Gamma}\left(q u-q_{i} u_{i}\right) \cdot \nabla_{\Gamma} v_{n}+\Delta_{\Gamma}\left(q u-q_{i} u_{i}\right) v_{n}\right] \\
= & \sum_{i=1}^{n_{q}} \int_{\Gamma_{i}} \partial_{n} p_{i} \partial_{n}\left(u-u_{i}\right) v_{n}+\int_{\Gamma_{i}} p q^{-1} \nabla_{\Gamma}\left(q u-q_{i} u_{i}\right) \cdot \nabla_{\Gamma} v_{n}-\nabla_{\Gamma}\left(q u-q_{i} u_{i}\right) \cdot \nabla_{\Gamma}\left(\frac{p}{q} v_{n}\right) \\
= & \sum_{i=1}^{n_{q}} \int_{\Gamma_{i}}\left[\partial_{n} p_{i} \partial_{n}\left(u-u_{i}\right)-\nabla_{\Gamma}\left(u-\frac{q_{i}}{q} u_{i}\right) \cdot \nabla_{\Gamma} p\right] v_{n} .
\end{aligned}
$$

According to the boundary conditions (4.1),(4.2) and (4.8) we get

$$
\begin{aligned}
d J\left(\left\{\Omega_{i}\right\}_{i=1}^{n_{q}} ; V\right) & =\sum_{i=1}^{n_{q}} \int_{\Gamma_{i}}\left(q_{i}-q\right)\left(\partial_{n} p_{i} \partial_{n} u+\nabla_{\Gamma} u \cdot \nabla_{\Gamma} p_{i}\right) v_{n}+\beta\left|q-q_{i}\right| \int_{\Gamma_{i}} \mathcal{H} v_{n} \\
& =\sum_{i=1}^{n_{q}} \int_{\Gamma_{i}}\left[\left(q_{i}-q\right)\left(\nabla p_{i} \cdot \nabla u\right)+\beta\left|q-q_{i}\right| \mathcal{H}\right] v_{n}
\end{aligned}
$$

4.2. Derivative with respect to the conductivity $q_{i}$. We consider a family of subregions and conductivities $\left\{\Omega_{i}, q_{i}\right\}_{i \in\left\{1, . ., n_{q}\right\}}$ as in section 4.1. The small perturbations are of the form $q_{i}^{\eta}=q_{i}+\eta q_{i}^{\prime}$, where $\eta$ is a small real parameter. Denote by $u^{\eta}$ and $u_{i}^{\eta}$ the solutions of (4.1)-(4.2) with $q^{\eta}$ instead of $q$. Formally, we define the derivatives $u^{\prime}$ and $u_{i}^{\prime}$ by

$$
u^{\prime}=\lim _{\eta \rightarrow 0} \frac{u^{\eta}-u}{\eta} \quad \text { and } \quad u_{i}^{\prime}=\lim _{\eta \rightarrow 0} \frac{u_{i}^{\eta}-u_{i}}{\eta} .
$$

Substituting (4.13) in (3.2)-(3.3) we get the following equations for $u^{\prime}$ and $u_{i}^{\prime}$ :

$$
\begin{aligned}
& -\Delta u^{\prime}=0 \text { in } \Omega^{*}, \quad q \partial_{n} u^{\prime}=0 \text { on } \Sigma, \quad q \partial_{n} u^{\prime}=q_{i}^{\prime} \partial_{n} u_{i}+q_{i} \partial_{n} u_{i}^{\prime} \text { on } \Gamma_{i}, \\
& -\Delta u_{i}^{\prime}=0 \text { in } \Omega_{i}, \quad u_{i}^{\prime}=u^{\prime} \text { on } \Gamma_{i} .
\end{aligned}
$$


We assume that $q_{i} \neq q$ for all $i$ as $q_{i}=q$ leads to a trivial situation. Therefore, the derivative of $\mathcal{J}$ with respect to $q_{i}$ is

$$
d \mathcal{J}\left(\left\{\Omega_{j}, q_{j}\right\}_{j=1}^{n_{q}} ; q_{i}^{\prime}\right)=\int_{\Sigma} 2(u-m) u^{\prime}+\operatorname{sign}\left(q_{i}-q\right) \beta q_{i}^{\prime} \mathcal{P}\left(\Gamma_{i}\right) .
$$

The first term on the right-hand side of (4.16) may be computed using the adjoint states $\left(p, p_{i}\right)$ defined in (4.7)-(4.8). From Green's formula for $p_{i}$ and $p$ in $\Omega_{i}$ and $\Omega^{*}$, respectively, we get

$$
\int_{\Sigma} 2(u-m) q^{-1} u^{\prime}=\int_{\Sigma} \partial_{n} p u^{\prime}=\int_{\Gamma_{i}}-u^{\prime} \partial_{n} p+u_{i}^{\prime} \partial_{n} p_{i}+p \partial_{n} u^{\prime}-p_{i} \partial_{n} u_{i}^{\prime}
$$

Finally, using the relations (4.14)-(4.15) and (4.7)-(4.8) and Green's formula in $\Omega_{i}$ we obtain

$$
d \mathcal{J}\left(\left\{\Omega_{j}, q_{j}\right\}_{j=1}^{n_{q}} ; q_{i}^{\prime}\right)=q_{i}^{\prime}\left(\operatorname{sign}\left(q_{i}-q\right) \beta q_{i}^{\prime} \mathcal{P}\left(\Gamma_{i}\right)-\int_{\Omega_{i}} \nabla u_{i} \cdot \nabla p_{i}\right) .
$$

5. Numerical results. When dealing with the topological derivative from a numerical viewpoint, we face several algorithmical possibilities. If the number of inclusions is unknown, one may have a conservative approach and look for one inclusion at a time. One computes the topological derivative and, according to the topological expansion, creates an inclusion whose center is located at the minimum of the topological derivative. This process is iterated until the topological derivative is positive all over the domain $\Omega$. It has been observed, though, that one may be much more aggressive and look for several inclusions in one iteration, by considering all negative values of the topological derivative, or a subset of it. This works well if the inclusions have similar sizes. Small inclusions will typically be "hidden" by larger inclusions. With our higher-order expansions, we can capture several inclusions in one step in a more efficient way. However, using the formula for multiple inclusions also increases the computational cost since more possibilities of inclusion positions must be explored. Therefore one has to find the right balance between this cost and efficiency of the method. In this paper, we provide some advice on how to use these additional terms.

When considering topological and non-smooth modifications of a shape, there is actually a vast amount of possibilities, which may not be considered in a single paper. The cases of different radii or shapes (including non-smooth shapes) for the simultaneous inclusions, and the case of several close or intersecting objects and inclusions very close to the border are all interesting cases which are not handled by our asymptotic expansions. Although asymptotic formulas may be obtained theoretically for these situations, one has to consider that they may not be practicable from a numerical point of view, as they would increase the computational load considerably. It is then better to have a rough estimate of the topology using the topological derivative and then to capture these features later with shape sensitivity.

In our numerical tests, we use $\Omega=(0,1) \times(0,1)$ and finite volumes to solve the state equation (4.1)-(4.2) and the adjoint equation (4.7)-(4.8) on a uniform grid of size $60 \times 60$.

5.1. Comparison of the topological derivative at different orders. In Figure 5.1, with the assumption $n_{q}=1$, we compare three different types of topological derivatives: the "classical" first-order topological derivative $\mathcal{T}_{0, \varepsilon}^{1}$, the extended topological derivative $\mathcal{T}_{0, \varepsilon}^{1}+L_{0}^{\varepsilon}$ considered in [15], and the full expansion (3.55) obtained in this paper given by $\mathcal{T}_{0, \varepsilon}^{1}+\mathcal{T}_{1, \varepsilon}^{1}+$ $\mathcal{T}_{2, \varepsilon}^{1,1}+L_{0}^{\varepsilon}$, under the assumption that $n_{q}=1$. We further assume that $q_{1}$ is known and take $M=8$ measurements. A range of three test values for $\varepsilon$ is chosen, with the middle value being the true size of the inclusion. In Figure 5.1, the inclusion is a ball of radius $\varepsilon=0.05$ and center $x_{1}=(0.35,0.3)$ and the topological derivatives are represented by the color plots, 

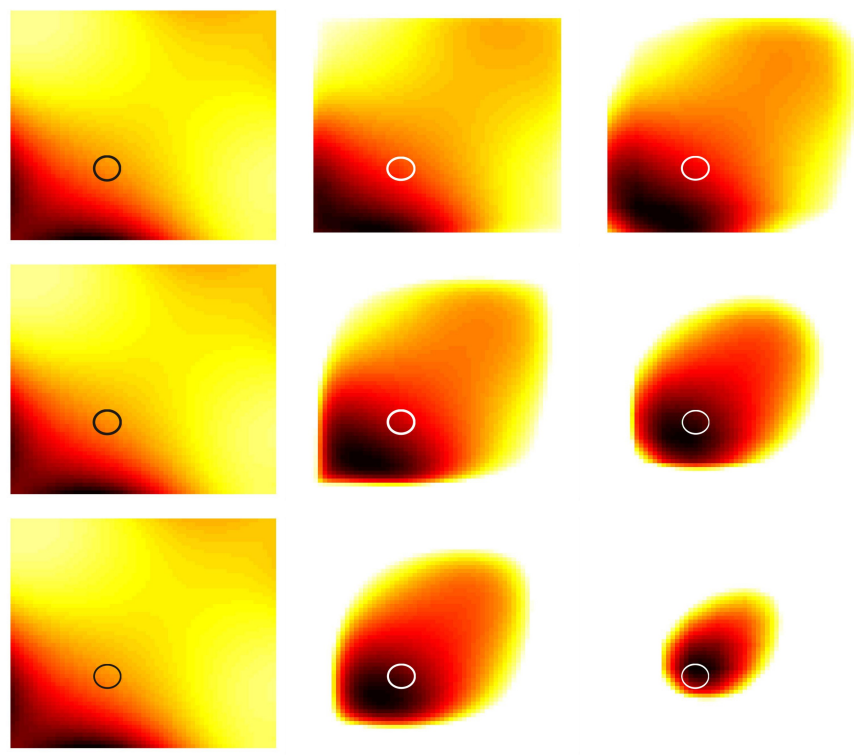

FIG. 5.1. Columns: $\mathcal{T}_{0, \varepsilon}^{1}$ (left), $\mathcal{T}_{0, \varepsilon}^{1}+L_{\varepsilon}^{0}$ (center), $\mathcal{T}_{0, \varepsilon}^{1}+\mathcal{T}_{1, \varepsilon}^{1}+\mathcal{T}_{2, \varepsilon}^{1,1}+L_{\varepsilon}^{0}$ (right). The lines correspond, from top to bottom, to three different trial values of $\varepsilon$ in ascending order, the middle line corresponding to the true value. Dark colors indicate negative values of the topological derivative.

where the darkest color corresponds to the lowest value. The contour of the true inclusions are the circles in the lower left corner of each plot.

In Figure 5.1, the columns correspond to the three different types of topological derivatives, while the lines correspond to the trial values of $\varepsilon$. For $\mathcal{T}_{0, \varepsilon}^{1}$ (left column), we observe the phenomenon which motivated our extended expansions in [15], i.e. the most negative values of the first-order term $\mathcal{T}_{0, \varepsilon}^{i}$ are always concentrated near the boundary $\Sigma$. This phenomenon is due to the non-uniform behavior of the remainder when considering only $\mathcal{T}_{0, \varepsilon}^{1}$. This substantiates the assumption that the centers of the inclusions have to be at a certain distance from the boundary $\Sigma$. Since the most negative value of the topological derivative indicates the creation of an inclusion, $\mathcal{T}_{0, \varepsilon}^{1}$ clearly provides the wrong result in all cases. Numerically, it was shown in [15] that $\mathcal{T}_{0, \varepsilon}^{1}+L_{0}^{\varepsilon}$ allows to obtain good results by correcting the topological derivative on the boundary of the domain $\Omega$. Such a correction can be seen in the second column of Figure 5.1. However, one observes a certain mismatch between the true value of $\varepsilon$ and the value indicated by the topological derivative. Indeed, the topological derivative provides a better estimate of the location when one chooses $\varepsilon$ larger than the true value of $\varepsilon$; see the plot in third line and second column. Finally, the last column of Figure 5.1 shows that this mismatch almost disappears when considering higher-order terms. For the true value of $\varepsilon$ (second line), the topological derivative is most negative close to the true location of the inclusion.

5.2. Combined topology and shape algorithm. We introduce the notation

$$
\mathcal{T}_{\varepsilon}\left(\left\{x_{k}\right\}_{k=1}^{n_{q}}\right)=\sum_{i=1}^{n_{q}}\left(\mathcal{T}_{0, \varepsilon}^{i}\left(x_{i}\right)+\mathcal{T}_{1, \varepsilon}^{i}\left(x_{i}\right)+\sum_{j=1}^{n_{q}} \mathcal{T}_{2, \varepsilon}^{i, j}\left(x_{i}, x_{j}\right)+\sum_{j \neq i} \mathcal{T}_{3, \varepsilon}^{i, j}\left(x_{i}, x_{j}\right)\right)+L_{0}^{\varepsilon}
$$

The usual procedure for applying the topological derivatives is to create a small inclusion where its minimum is attained. In the case of higher-order expansions, the topological deriva- 
tive depends on $\varepsilon$. Thus, a possible approach is to minimize first $\mathcal{T}_{\varepsilon}$ with respect to $\varepsilon$, and then with respect to the position. We choose a given range for the minimization with respect to $\varepsilon$, i.e. we choose an interval $\left[\varepsilon_{0}, \varepsilon_{1}\right]$, and we define the following function

$$
\mathcal{T}_{m}\left(\left\{x_{k}\right\}_{k=1}^{n_{q}}\right):=\min _{\varepsilon \in\left[\varepsilon_{0}, \varepsilon_{1}\right]} \mathcal{T}_{\varepsilon}\left(\left\{x_{k}\right\}_{k=1}^{n_{q}}\right) \text {. }
$$

In the numerical computation, the interval $\left[\varepsilon_{0}, \varepsilon_{1}\right]$ is discretized into a user-defined number of values. This discretization may be very fine and does not impose a heavy computational load.

There are two approaches of how to use $\mathcal{T}_{m}\left(\left\{x_{k}\right\}_{k=1}^{n_{q}}\right)$ : (1) the first approach consists in finding the minimum of $\mathcal{T}_{m}\left(\left\{x_{k}\right\}_{k=1}^{n_{q}}\right)$ and in obtaining a set of points $\left\{\bar{x}_{k}\right\}_{k=1}^{n_{q}}$. Then $\varepsilon$ is given by the minimizing argument in (5.2). Hence, the domain is initialized by creating inclusions with centers $\left\{\bar{x}_{k}\right\}_{k=1}^{n_{q}}$ and respective radius $\varepsilon$ (the shape of inclusions are balls, but "any" shape could be considered instead). Note that our computations may be extended to the case of a set of different radii $\left\{\varepsilon_{k}\right\}_{k=1}^{n_{q}}$. (2) The second approach consists in choosing a threshold $\gamma$ and in initializing the domain $\Omega_{1}$ as

$$
\Omega_{1}:=\left\{x \in \Omega \mid \mathcal{T}_{m}(x)<\gamma \min _{y \in \Omega} \mathcal{T}_{m}(y)\right\}
$$

The first approach is completely automatized, but allows only to create inclusions with fixed shapes. The second approach is more flexible in terms of the shape, and may therefore provide finer results. But it needs a user-defined parameter $\gamma$ as an input quantity. In [15] we relied on the second approach. In this paper we focus on the first one.

After this initialization by topological sensitivity, the remaining iterations are as follows: First we update the shape using the shape derivative in a steepest descent framework. According to (4.12) the steepest descent direction is given by

$$
v_{n}=-\left[\left(q_{i}-q\right)\left(\nabla p_{i} \cdot \nabla u\right)+\beta\left|q-q_{i}\right| \mathcal{H}\right] \quad \text { on } \Gamma_{i} .
$$

For the representation and transport of the geometry of the $\Omega_{i}$ according to the steepest descent direction, we use a level set method [20,21]. Note that due to the nature of shape sensitivity, the update velocity $v_{n}$ normal to the internal boundary of $\Omega$ is defined only on the $\Gamma_{i}$ 's. As the transport of domains is achieved by the level set equation $\phi_{t}+V\|\nabla \phi\|=0$ in $\Omega$, we have to extend $v_{n}$ to the entire domain $\Omega$ (this yields $V$ in the above equation). Above, $\phi$ represents the level set function which we assume to be a signed distance function with $\left.\phi\right|_{\Gamma}=0$. We extend $v_{n}$ constant along normals to $\Gamma$. Observe that the level set equation is a partial differential equation of Hamilton-Jacobi-type. For its appropriate numerical treatment we refer to [20, 21]. Having advanced the geometry, in a second step, according to (4.18), the value of $q_{i}$ is updated along the direction

$$
q_{i}^{\prime}=-\operatorname{sign}\left(q_{i}-q\right) \beta q_{i}^{\prime} \mathcal{P}\left(\Gamma_{i}\right)+\int_{\Omega_{i}} \nabla u_{i} \cdot \nabla p_{i}
$$

again within a steepest descent framework. For choosing an appropriate step length, we use a standard Armijo line search procedure with backtracking.

In [9], a different method, also based on a levelset representation of the domain, is used. It relies on the use of a smoothed Heaviside function, evaluated at the level set function, to represent the domain. The level set function itself is then used as the "shape variable". On one hand, the method has the advantage to be easy to handle and to be able to provide both topological and shape changes. On the other hand, it relies on the use of an arbitrary smoothing parameter of the Heavyside function whose appropriate choice is crucial for the correct evolution of the process. Therefore, the method is more heuristic and not theoretically supported 

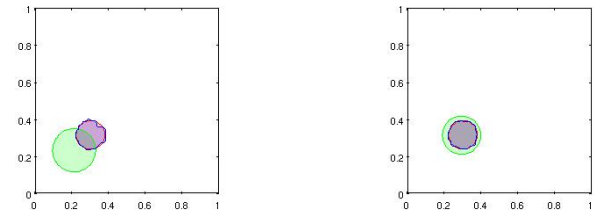

FIG. 5.2. Red: true inclusion. Green: initialization. Blue: final result. After 300 iterations using $\mathcal{T}_{0, \varepsilon}+L_{\varepsilon}^{0}$ (left), after 50 iterations using $\mathcal{T}_{0, \varepsilon}+\mathcal{T}_{1, \varepsilon}+\mathcal{T}_{2, \varepsilon}+L_{\varepsilon}^{0}$ (right)
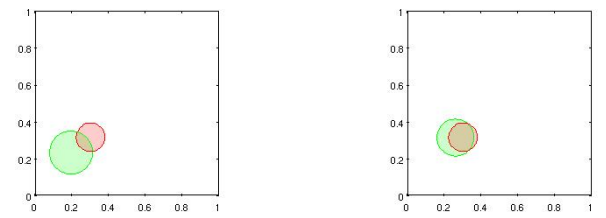

FIG. 5.3. Initialization with $5 \%$ noise. Red: true inclusion. Green: initialization. Using $\mathcal{T}_{0, \varepsilon}+L_{\varepsilon}^{0}$ (left). Using $\mathcal{T}_{0, \varepsilon}+\mathcal{T}_{1, \varepsilon}+\mathcal{T}_{2, \varepsilon}+L_{\varepsilon}^{0}($ right $)$

by an asymptotic and shape analysis as in our method. It would be interesting to compare the two methods numerically, but also analytically for vanishing smoothing parameter of the Heaviside function, and to establish possible parallels between them.

We point out that the shape as well as the $q$-update procedure might rely on Newton-type updates as well. This, however, requires the additional work of solving elliptic systems on the $\Gamma_{i}$ 's and is subject to future research.

5.3. One inclusion. In the case of one inclusion $\left(n_{q}=1\right)$, in Figure 5.2 we compare the results obtained by using the first-order expansion and corrections $\mathcal{T}_{0, \varepsilon}^{1}+L_{0}^{\varepsilon}$, and the higherorder expansion given by (5.1), respectively. We use the first approach for the topological derivative in Figure 5.2. To reduce the amount of computations, the function $h$ is computed only on a subgrid of size $10 \times 10$, and then it is interpolated on the finer grid $60 \times 60$. The number of measurements is $M=2$. The range for the $\varepsilon$-minimization in (5.2) is $\left[\varepsilon_{0}, \varepsilon_{1}\right]=$ $(0.02,0.12)$, and $\left[\varepsilon_{0}, \varepsilon_{1}\right]$ is split into 60 equal intervals in our numerical tests. The true values for the conductivities are $q=10$ and $q_{1}=1$.

In Figure 5.2, the true inclusion, highlighted in red, is a ball of radius $\varepsilon_{1}=0.08$ and position $x_{1}=(0.31,0.3)$. The initial guess provided by the topological derivative is the green ball, and the blue object is the final result, after the application of the shape derivative part of the algorithm. The plot on the left is the result using the first-order expansion while the graph on the right is the result using higher-order topological expansions. One clearly finds that the initialization provided by the first-order expansion and corrections, i.e. $\mathcal{T}_{0, \varepsilon}^{1}\left(x_{1}\right)+$ $L_{0}^{\varepsilon}$, is not as good as the one provided by higher-order topological expansions, with respect to both position and inclusion size. This slows down the shape optimization part of our algorithm: 300 iterations for first-order expansion instead of 50 for higher-order expansions. The reconstruction is also slightly worse in the case of first-order expansion although the ball is rather well-reconstructed in both cases.

In Figure 5.3, the same data as in Figure 5.2 are used, respectively, but with 5\% noise added to the measurements. Comparing the initializations obtained from topological sensitivity, it can be seen that the initialization using the topological derivative is rather robust for both types (first and higher-order).

In Figure 5.4 we study the case where $q$ is known and $q_{1}$ is unknown. The inclusion is a 

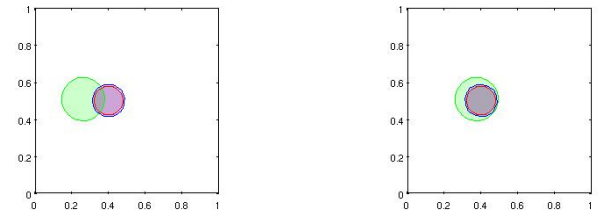

FIG. 5.4. Red: true inclusion. Green: initialization. Blue: final result. Using $\mathcal{T}_{0, \varepsilon}+L_{\varepsilon}^{0}$ (left). Using $\mathcal{T}_{0, \varepsilon}+\mathcal{T}_{1, \varepsilon}+\mathcal{T}_{2, \varepsilon}+L_{\varepsilon}^{0}$ (right)

ball of radius $\varepsilon_{1}=0.08$ and position $x_{1}=(0.4,0.5)$, the true value of $q_{1}$ is 2 . We start with the initial guess $q_{1}^{i n}=3$ and obtain the final value $q_{1}^{f i}=2.065$ after convergence (500 iterations for $q_{1}$ and 500 iterations for the shape) using the first-order topological derivative for the initialization and $q_{1}^{f i}=1.991$ after convergence ( 150 iterations for $q_{1}$ and 150 iterations for the shape) using the higher-order topological derivative for the initialization. We observe that in both cases, the initialization is robust with respect to $q_{1}$.

The conclusion from these numerical tests for one inclusion is that the initialization using the topological derivative is robust with respect to noise and unknown conductivity, and the higher-order topological derivative gives a better initial guess and helps to speed up the convergence of the shape and conductivity optimization part of the algorithm.

5.4. Two inclusions. In this section we reconstruct two objects, i.e. $n_{q}=2$. Numerically, there are several difficulties when $n_{q}>1$. First, the expansions that we have computed previously are not valid when the objects get too close to each other. Thus, the trial points $x_{k}, k=1, . ., n_{q}$ should be sufficiently distant from each other; otherwise we may have a significant error in the topological derivative which may lead to wrong initial guesses. Mathematically, this requires to ensure a minimal distance $d_{0}>0$ with $d\left(x_{k}, x_{l}\right)>d_{0}$ for all $k \neq l, k, l=1, . ., n_{q}$. The radius $\varepsilon$ should also guarantee that different balls have no intersection and remain at a certain distance from each other. For this purpose we solve (5.2) on a subgrid of the mesh, where we compute $u$ and $p$. In this way we ensure automatically that the points $x_{k}, k=1, . ., n_{q}$ have a certain mutual distance (the case $x_{k_{1}}=x_{k_{2}}$ is of course forbidden). Therefore, when the subgrid increases, on one hand the asymptotic expansions become more inaccurate, whereas on the other hand, the position becomes more accurate. Thus one has to find a balance between these two opposing behaviors.

The number of measurements is $M=4$. In Figure 5.5, we compare the results for different subgrids (dashed lines) of the main grid of size $60 \times 60$ for computing $u$. In our tests, we choose two levels of subgrids: $5 \times 5$ and $7 \times 7$. The two first columns correspond to the subgrid $5 \times 5$ and the third column to the subgrid $7 \times 7$. We find that both subgrids provide good results, up to their respective accuracy. Therefore, a coarse subgrid might provide a better result than a fine subgrid if the inclusions are by chance aligned on this coarse subgrid; see for instance the first row in Figure 5.5. In the two first rows, the two inclusions have the same sizes, whereas in the third row, the two inclusions have different sizes. The reconstruction might fail when the two inclusions have different sizes. This is due to the stronger influence of the bigger object on the measurements. Studying the influence of different inclusion sizes on the reconstruction process raises several interesting questions, which, however, are beyond the scope of the present paper. We use the same positions for the three Figures, only the sizes are changed. In the two first lines the sizes are $\varepsilon=0.05$ and $\varepsilon=0.08$, respectively, and in the third line the sizes are $\varepsilon_{1}=0.05$ and $\varepsilon_{2}=0.07$.

Acknowledgement. The authors acknowledge financial support by the Austrian Ministry of Science and Education and the Austrian Science Fundation FWF under START-grant 

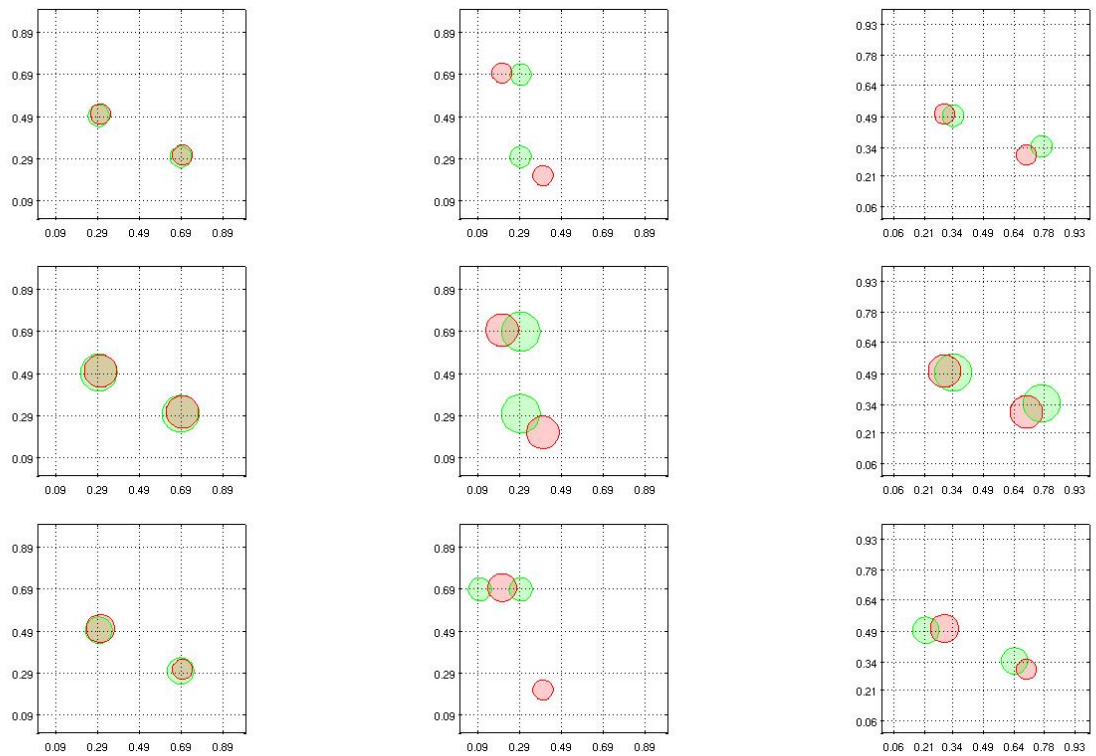

FIG. 5.5. First and second columns: subgrid $5 \times 5$. Third column: subgrid $7 \times 7$. True location of inclusions (red), initial guess by topological derivative (green). Subgrids represented by dashed lines. First and third column: $\left(x_{1}, y_{1}\right)=(0.7,0.3),\left(x_{2}, y_{2}\right)=(0.3,0.5)$. Second column: $\left(x_{1}, y_{1}\right)=(0.4,0.2),\left(x_{2}, y_{2}\right)=(0.2,0.7)$.

Y305 "Interfaces and free boundaries" and the subproject "Freelevel" of the SFB F32 "Mathematical Optimization and Applications in Biomedical Sciences".

\section{REFERENCES}

[1] H. Ammari and H. Kang. High-order terms in the asymptotic expansions of the steady-state voltage potentials in the presence of conductivity inhomogeneities of small diameter. SIAM J. Math. Anal., 34(5):11521166 (electronic), 2003.

[2] H. Ammari, S. Moskow, and M. S. Vogelius. Boundary integral formulae for the reconstruction of electric and electromagnetic inhomogeneities of small volume. ESAIM Control Optim. Calc. Var., 9:49-66, 2003.

[3] V. Bonnaillie-Noël, M. Dambrine, S. Tordeux, and G. Vial. On moderately close inclusions for the Laplace equation. C. R. Math. Acad. Sci. Paris, 345(11):609-614, 2007.

[4] M. Bonnet. Higher-order topological sensitivity for 2-D potential problems. Application to fast identification of inclusions. Internat. J. Solids Structures, 46(11-12):2275-2292, 2009.

[5] L. Borcea. Electrical impedance tomography. Inverse Problems, 18(6):R99-R136, 2002.

[6] M. Brühl, M. Hanke, and M. S. Vogelius. A direct impedance tomography algorithm for locating small inhomogeneities. Numer. Math., 93(4):635-654, 2003.

[7] D. J. Cedio-Fengya, S. Moskow, and M. S. Vogelius. Identification of conductivity imperfections of small diameter by boundary measurements. Continuous dependence and computational reconstruction. Inverse Problems, 14(3):553-595, 1998.

[8] M. Cheney, D. Isaacson, and J. C. Newell. Electrical impedance tomography. SIAM Rev., 41(1):85-101 (electronic), 1999.

[9] E. T. Chung, T. F. Chan, and X.-C. Tai. Electrical impedance tomography using level set representation and total variational regularization. J. Comput. Phys., 205(1):357-372, 2005.

[10] J. R. de Faria, A. A. Novotny, R. A. Feijóo, E. Taroco, and C. Padra. Second order topological sensitivity analysis. Internat. J. Solids Structures, 44(14-15):4958-4977, 2007.

[11] M. C. Delfour and J.-P. Zolésio. Shapes and geometries, volume 4 of Advances in Design and Control. Society for Industrial and Applied Mathematics (SIAM), Philadelphia, PA, 2001. 
[12] H. Eschenauer, V. Kobelev, and A. Schumacher. Bubble method for topology and shape optimization of structures. 8:42-51, 1994.

[13] S. Garreau, P. Guillaume, and M. Masmoudi. The topological asymptotic for PDE systems: the elasticity case. SIAM J. Control Optim., 39(6):1756-1778 (electronic), 2001.

[14] A. Henrot. Extremum problems for eigenvalues of elliptic operators. Frontiers in Mathematics. Birkhäuser Verlag, Basel, 2006.

[15] M. Hintermüller and A. Laurain. Electrical impedance tomography: from topology to shape. Control Cybernet., 37(4):913-933, 2008.

[16] A. M. Il'in. Matching of asymptotic expansions of solutions of boundary value problems, volume 102 of Transl. Math. Monog. American Mathematical Society, Providence, RI, 1992.

[17] W. G. Mazja, S. A. Nasarow, and B. A. Plamenewski. Asymptotische Theorie elliptischer Randwertaufgaben in singulär gestörten Gebieten. I, volume 82 of Mathematische Lehrbücher und Monographien, II. Abteilung: Mathematische Monographien. Akademie-Verlag, Berlin, 1991.

[18] S. A. Nazarov and J. Sokolowski. Spectral problems in shape optimization. Singular boundary perturbations. Asymptotic Analysis, 56(3-4):159-196, 2008.

[19] S. A. Nazarov and J. Sokolowski. Shape sensitivity analysis of eigenvalues revisited. Control and Cybernetics, 37(4):999-1012, 2008.

[20] S. Osher and R. Fedkiw. Level set methods and dynamic implicit surfaces, volume 153 of Applied Mathematical Sciences. Springer-Verlag, New York, 2003.

[21] S. Osher and J. A. Sethian. Fronts propagating with curvature-dependent speed: algorithms based on Hamilton-Jacobi formulations. J. Comput. Phys., 79(1):12-49, 1988.

[22] J. Sokołowski and A. Żochowski. On the topological derivative in shape optimization. SIAM J. Control Optim., 37(4):1251-1272 (electronic), 1999.

[23] J. Sokołowski and J.-P. Zolésio. Introduction to shape optimization, volume 16 of Springer Series in Computational Mathematics. Springer-Verlag, Berlin, 1992. Shape sensitivity analysis.

6. Appendix. For vectors $a$ and $b$, let us compute the integral

$$
\mathcal{I}=\int_{\partial B_{i}^{\varepsilon}}(a \cdot n)(b \cdot n) .
$$

Since $(a \otimes b) c=(b \cdot c) a$ and $\int_{\partial B_{i}^{\varepsilon}} n \otimes n=\varepsilon^{-1}\left|B_{i}^{\varepsilon}\right| I$, we have

$$
\mathcal{I}=(a \otimes b) \cdot \int_{\partial B_{i}^{\varepsilon}} n \otimes n=\frac{\left|B_{i}^{\varepsilon}\right|}{\varepsilon}(a \otimes b) \cdot I=\frac{\left|B_{i}^{\varepsilon}\right|}{\varepsilon} a \cdot b .
$$

By setting $a=\nabla u^{0}\left(x_{i}\right)$ and $b=\nabla p\left(x_{i}\right)$ we obtain

$$
\int_{\partial B_{i}^{\varepsilon}}\left(\nabla u^{0}\left(x_{i}\right) \cdot n\right)\left(\nabla p\left(x_{i}\right) \cdot n\right)=\frac{\left|B_{i}^{\varepsilon}\right|}{\varepsilon} \nabla u^{0}\left(x_{i}\right) \cdot \nabla p\left(x_{i}\right) .
$$

For $A=A^{T}$ and $B=B^{T}$ second-order tensors, let us take into account the integral

$$
\mathcal{I}=\int_{\partial B_{i}^{\varepsilon}}(A n \cdot n)(B n \cdot n) .
$$

Since $(A \otimes B) C=(B \cdot C) A$ and $\int_{\partial B_{i}^{\varepsilon}}(n \otimes n \otimes n \otimes n)=\varepsilon^{-1}(N+2)^{-1}\left|B_{i}^{\varepsilon}\right|(2 I I+I \otimes I)$, where $I$ is the fourth-order identity tensor. Then

$$
\begin{aligned}
\mathcal{I}=\int_{\partial B_{i}^{\varepsilon}}(n \otimes n \otimes n \otimes n) A \cdot B & =\frac{\left|B_{i}^{\varepsilon}\right|}{\varepsilon(N+2)}(2 I I+I \otimes I) A \cdot B \\
& =\frac{\left|B_{i}^{\varepsilon}\right|}{\varepsilon(N+2)}(2 A \cdot B+\operatorname{tr}(A) \operatorname{tr}(B)) .
\end{aligned}
$$


By setting $A=D^{2} u^{0}\left(x_{i}\right)$ and $B=D^{2} p\left(x_{i}\right)$ we obtain

$$
\begin{aligned}
\int_{\partial B_{i}^{\varepsilon}} D^{2} u^{0}\left(x_{i}\right)(n)^{2} D^{2} p\left(x_{i}\right)(n)^{2} & =\frac{\left|B_{i}^{\varepsilon}\right|}{\varepsilon(N+2)}\left(2 D^{2} u^{0}\left(x_{i}\right) \cdot D^{2} p\left(x_{i}\right)+\operatorname{tr}\left(D^{2} u^{0}\left(x_{i}\right)\right) \operatorname{tr}\left(D^{2} p\left(x_{i}\right)\right)\right) \\
& =\frac{\left|B_{i}^{\varepsilon}\right|}{\varepsilon(N+2)}\left(2 D^{2} u^{0}\left(x_{i}\right) \cdot D^{2} p\left(x_{i}\right)+\Delta u^{0}\left(x_{i}\right) \Delta p\left(x_{i}\right)\right) .
\end{aligned}
$$

For $C$ third-order tensor and $a$ vector, let us compute the integral

$$
\mathcal{I}=\int_{\partial B_{i}^{\varepsilon}}((C n) n \cdot n)(a \cdot n)
$$

By setting $C=D^{3} p\left(x_{i}\right)$ and $a=\nabla u^{0}\left(x_{i}\right)$, we have

$$
\int_{\partial B_{i}^{\varepsilon}} D^{3} p\left(x_{i}\right)(n)^{3} \nabla u^{0}\left(x_{i}\right) \cdot n=c_{\varepsilon} \nabla\left(\Delta p\left(x_{i}\right)\right) \cdot \nabla u^{0}\left(x_{i}\right)
$$

with $c_{\varepsilon}=\frac{3}{4} \pi \varepsilon$ for $N=2$ and $c_{\varepsilon}=\frac{3}{5} \frac{4}{3} \pi \varepsilon^{2}$ for $N=3$. Therefore

$$
\int_{\partial B_{i}^{\varepsilon}} D^{3} p\left(x_{i}\right)(n)^{3} \nabla u^{0}\left(x_{i}\right) \cdot n=\frac{3}{(N+2)} \frac{\left|B_{i}^{\varepsilon}\right|}{\varepsilon} \nabla\left(\Delta p\left(x_{i}\right)\right) \cdot \nabla u^{0}\left(x_{i}\right) .
$$

Finally, by setting $C=D^{3} u^{0}\left(x_{i}\right)$ and $a=\nabla p\left(x_{i}\right)$, we have

$$
\int_{\partial B_{i}^{\varepsilon}} D^{3} u^{0}\left(x_{i}\right)(n)^{3} \nabla p\left(x_{i}\right) \cdot n=\frac{3}{(N+2)} \frac{\left|B_{i}^{\varepsilon}\right|}{\varepsilon} \nabla\left(\Delta u^{0}\left(x_{i}\right)\right) \cdot \nabla p\left(x_{i}\right) .
$$

